\title{
Article \\ Applicability and Limitations of Simplified Elastic Shell Theories for Vibration Modelling of Double-Walled Carbon Nanotubes
}

\author{
Matteo Strozzi ${ }^{1,2, *(\mathbb{D})}$, Oleg V. Gendelman ${ }^{2}$, Isaac E. Elishakoff ${ }^{3}$ and Francesco Pellicano ${ }^{4}(\mathbb{D}$ \\ 1 Department of Sciences and Methods for Engineering, University of Modena and Reggio Emilia, \\ 42122 Reggio Emilia, Italy \\ 2 Faculty of Mechanical Engineering, Technion, Israel Institute of Technology, Haifa 32000, Israel; \\ ovgend@me.technion.ac.il \\ 3 Department of Ocean and Mechanical Engineering, Florida Atlantic University, Boca Raton, FL 33431, USA; \\ elishako@fau.edu \\ 4 Department of Engineering “Enzo Ferrari”, Centre InterMech Mo.Re., University of Modena and Reggio \\ Emilia, 41125 Modena, Italy; francesco.pellicano@unimore.it \\ * Correspondence: matteo.strozzi@unimore.it
}

Citation: Strozzi, M.; Gendelman, O.V.; Elishakoff, I.E.; Pellicano, F. Applicability and Limitations of Simplified Elastic Shell Theories for Vibration Modelling of Double-Walled Carbon Nanotubes. C 2021, 7, 61. https://doi.org/10.3390/c7030061

Academic Editor: Gil Goncalves

Received: 2 July 2021

Accepted: 4 August 2021

Published: 9 August 2021

Publisher's Note: MDPI stays neutral with regard to jurisdictional claims in published maps and institutional affiliations.

Copyright: () 2021 by the authors. Licensee MDPI, Basel, Switzerland. This article is an open access article distributed under the terms and conditions of the Creative Commons Attribution (CC BY) license (https:// creativecommons.org/licenses/by/ $4.0 /)$.

\begin{abstract}
The applicability and limitations of simplified models of thin elastic circular cylindrical shells for linear vibrations of double-walled carbon nanotubes (DWCNTs) are considered. The simplified models, which are based on the assumptions of membrane and moment approximate thin-shell theories, are compared with the extended Sanders-Koiter shell theory. Actual discrete DWCNTs are modelled by means of couples of concentric equivalent continuous thin, circular cylindrical shells. Van der Waals interaction forces between the layers are taken into account by adopting He's model. Simply supported and free-free boundary conditions are applied. The Rayleigh-Ritz method is considered to obtain approximate natural frequencies and mode shapes. Different aspect and thickness ratios, and numbers of waves along longitudinal and circumferential directions, are analysed. In the cases of axisymmetric and beam-like modes, it is proven that membrane shell theory, differently from moment shell theory, provides results with excellent agreement with the extended Sanders-Koiter shell theory. On the other hand, in the case of shell-like modes, it is found that both membrane and moment shell theories provide results reporting acceptable agreement with the extended Sanders-Koiter shell theory only for very limited ranges of geometries and wavenumbers. Conversely, for shell-like modes it is found that a newly developed, simplified shell model, based on the combination of membrane and semi-moment theories, provides results in satisfactory agreement with the extended Sanders-Koiter shell theory in all ranges.
\end{abstract}

Keywords: carbon nanotubes; double-walled; linear vibrations; Sanders-Koiter theory; simplified shell models

\section{Introduction}

Thin-walled mechanical components, such as beams, plates and shells, are adopted as structural elements in several engineering fields, in particular mechanical, aeronautical and aerospace, due to their specific mechanical properties, such as high strength and stiffness, low weight and light inertia.

As an example, monolithic rectangular tanks are used as engineering structures in sewage and water treatment plants, and recreational facilities. In most cases they are made with constant thickness walls, but adoption of variable thickness (e.g., trapezoidal crosssection) walls is justified in structures where hydrostatic pressure acts on the walls [1,2].

On the other hand, examples of thin shell structures adopted in mechanical systems are piping components, turbine disks, pressure vessels and liquid-retaining tanks, while 
aircrafts, missiles and rockets represent some examples of thin shell structure applications in aeronautical and aerospace field [3].

The wide application of thin shell structures in engineering field is due to their very high load-carrying capability, strength-to-weight ratio and structural stiffness. In particular, thin shell structures are able to efficiently support applied external forces by virtue of their characteristic spatial curvature, and, as a result, thin shells are much stronger and stiffer than all other structural forms [4].

The simplest, and therefore most adopted theories, to model shell behaviour are the elastic shell theories. These theories predict, adequately, both stresses and deformations for shells exhibiting elastic deformations, i.e., deformations for which Hooke's law holds into the equilibrium equations [5]. On the other hand, these theories are limited to elastic, i.e., reversible, deformations of shells and therefore they cannot be adopted in the case of structural fracture or failure [6].

An actual shell is a three-dimensional body; by using Kirchhoff-Love's assumptions [7], the three-dimensional problem of shell equilibrium and straining reduces to a two-dimensional problem at the shell middle surface. Therefore, in thin elastic shell theories, the shell problem is reduced to the study of the middle surface deformations, i.e., strains and changes in curvature and torsion [8].

Several thin elastic shell theories with varying degrees of accuracy were derived in literature, depending on the degree of simplification of the elasticity equilibrium equations. The approximations necessary for the development of a simplified but adequate thin elastic shell theory have been subject of relevant studies, performed in the past, by eminent elastic continuum mechanics scientists.

Love was the first to develop an approximate but accurate thin shell theory based on classical linear elasticity [9]. In order to simplify the strain-displacement relationships, and therefore the expression of the elastic strain energy, Love applied to the shell theory the same hypotheses that were originally developed by Kirchhoff for the plate bending theory, together with the small deflection and thin shell hypotheses. These assumptions are commonly called "Kirchhoff-Love's assumptions", and Love's theory of thin elastic shells is also referred to as "first-order approximation shell theory" [10].

After Love, many researchers tried to eliminate some inadequacies of his approximate theory, with particular regard to its inconsistent treatment of small terms into the equilibrium equations. The need for a mathematically rigorous two-dimensional set of shell equations adopting Kirchhoff-Love's assumptions led to different versions of the first-order approximation shell theory.

Reissner [11] developed a first-order approximation theory by deriving equilibrium, strain-displacement and stress resultant equations for thin shells directly from the threedimensional elasticity theory. Reissner's linear theory of thin elastic shells considers Kirchhoff-Love's assumptions and neglects the distance of the arbitrary point of the shell from the middle surface with respect to the radii of curvature of the shell middle surface.

Sanders [12] also developed a first-order approximation theory starting from the principle of virtual work and applying Kirchhoff-Love's assumptions. Sanders linear theory of thin elastic shells, which also removes inconsistencies of Love theory in small terms, was later modified and refined by Koiter, giving rise to Sanders-Koiter shell theory [13].

Donnell [14], Vlasov [15] and Mushtari [16], independently, developed a simplified thin shell theory assuming, besides Kirchhoff-Love's hypotheses, infinitesimal tangential displacements along longitudinal and circumferential directions into the straindisplacement relationships and equilibrium equations. Due to their simplicity, the governing equations of Donnell-Vlasov-Mushtari shell theory were found to be extremely convenient for solving many engineering problems related to shells.

Other first-order approximation shell theories, close to Love theory and considering Kirchhoff-Love's assumptions, were developed by Timoshenko [17] and Naghdi [18].

Besides first-order approximation shell theories, a second class of thin elastic shell theories, which are commonly referred to as higher-order approximation shell theories, 
was developed. To this second class they are assigned all thin elastic shell theories in which at least one of Kirchhoff-Love's assumptions is not respected.

Lur'ye [19], Flugge [20] and Byrne [21], independently of each other, developed a second-order approximation shell theory deriving equations of equilibrium, straindisplacement relationships and stress resultants from the application of Kirchhoff's hypotheses and small deflection assumption to the equations of the three-dimensional theory of elasticity, while removing the thin shell assumption.

Novozhilov [22] developed another version of the second-order approximation shell theory by deriving the strain-displacement relationships from the three-dimensional theory of elasticity by applying Kirchhoff's assumptions and the stress resultant equations from the strain energy expression, again removing, similarly to Lur'ye-Flugge-Byrne theory, thin shell assumption.

Other second-order approximation theories, obtained applying Kirchhoff-Love's assumptions but removing the thinness one, were developed also by Gol'denveizer [23] and Reissner [24].

Since the governing equations of the general thin elastic shell theories are eight-order partial differential equations, then the computation of the shell stress and strain components is very difficult. As a consequence, researchers involved in thin shell stress-strain analysis were focused on the simplification of the governing partial differential equations of shells.

The aforementioned thin elastic shell theories assumed that the bending (flexural) stresses were of the same order of the stretching (extensional) stresses. If one of the above-mentioned types of stresses is negligible, in comparison with the other one, then it is possible to obtain a considerable simplification in the characteristic equations and relationships of the shell theory, in the form of a simplified (reduced) elastic shell model.

The first possible type of stress state for thin elastic shells is the membrane (momentless) state, which is present if the bending stresses are negligible compared with the stretching ones $[25,26]$. The governing equations of the membrane shell theory can be derived directly from the equations of the general shell theory by neglecting bending and torsional moments on the stress state of the thin shell. The membrane shell theory is able to describe the state of stress with reasonable accuracy in the case of relatively short and very thin shells, when bending and torsional deformations are very small, while stretching deformations are preeminent, i.e., pure membrane state of stress.

The second possible type of stress state for thin elastic shells is the moment (forceless) state, which is present if the stretching stresses are negligible compared with the bending ones $[27,28]$. The governing equations of the moment shell theory can be obtained directly from the equations of the general shell theory by neglecting stretching (extensional) forces on the stress state of the thin shell. Contrary to membrane shell theory, moment shell theory is able to describe the state of stress with reasonable accuracy in the case of very long and relatively thick shells, when stretching deformations are very small, while bending and torsional deformations are preeminent, i.e., pure moment state of stress.

Research in nanotechnology has attracted numerous investigators in recent years. Recent status is reported in the monographs written by Elishakoff et al. [29] and Liew et al. [30], as well as in the volumes edited by Tserpes and Silvestre [31] and Harik [32].

Carbon nanotubes (CNTs) are used as ultrahigh frequency resonators in a large number of nano-electro-mechanical devices such as sensors, oscillators, charge detectors and field emission devices $[33,34]$. The reduction of the size and the increment of the stiffness of a nano-mechanical resonator magnify its resonant frequencies and decrease its energy consumption, therefore improving its sensitivity.

A relevant issue of CNTs is represented by their geometry, in particular their crosssection, which is important for the obtained results. Actually, for the practical CNT based composites, most of CNTs are compressed or collapsed to achieve a very dense packing. This specific CNT configuration, which presents a non-circular cross section, is beneficial for improving performance: in fact, collapsed carbon nanotubes represent ultralight structural 
materials with very high specific strength and stiffness comparable to current carbon fibre reinforced polymer composites, and with ultrahigh thermal conductivity [35-37].

A large number of experiments were conducted on single-walled carbon nanotubes (SWCNTs) [38-40]. In these works, the linear vibrations of free-free SWCNTs are analysed by adopting resonant Raman spectroscopy with laser excitation wavelengths in the range of nanometres. Several Raman peaks are observed, which correspond to the vibrational modes of the CNTs, and the CNT chirality is univocally assigned by measuring one radial breathing mode (RBM) frequency and applying the theory of the resonant transitions.

Because of their nanoscale size, it is very difficult to investigate the mechanical properties of the nanotubes adopting experimental techniques, which require the use of high resolution transmission electron microscopes and do not allow the natural frequencies of the different vibration modes to be easily separated within the frequency spectrum.

On the other hand, it was found that molecular dynamics (MD) simulations provide good predictions of the mechanical behaviour of SWCNTs and multi-walled carbon nanotubes (MWCNTs) under external forces, with results close to the experiments [41-43]. In these works, natural frequencies of RBMs and beam-like modes (BLMs) considering CNTs with different geometries, boundary conditions and chirality are computed adopting the molecular mechanics (MM) potential.

However, it has been demonstrated that MD simulations are computationally inefficient, especially when dealing with MWCNTs incorporating a large number of atoms. Therefore, more efficient analytical and numerical methods have been recently developed in order to analyse classes of CNTs in a more general and efficient way; such methods are generally based on continuous models for the nanotube and allow a strong reduction of the number of degrees of freedom. In particular, it was found that continuous thin elastic shell models can correctly predict static buckling and free vibration behaviour of CNTs obtained from experiments and MD simulations [44-48].

The analogies between thin elastic shell model and CNT structure led to an extensive adoption of thin elastic shell theories for CNT mechanical deformation analysis: applicability and limitations of thin elastic shell theories for vibration modelling of CNTs were studied by considering Love [49], Donnell-Mushtari [50-52], Sanders-Koiter [53,54] and Flügge-Byrne-Lur'ye $[55,56]$ theories.

Refs. [57-61] demonstrated the excellent agreement of the natural frequencies of SWCNTs and MWCNTs obtained by considering Sanders-Koiter shell theory with the results of experimental studies and MD simulations, for a large range of geometries and wavenumbers. Therefore, in the present work, the Sanders-Koiter shell theory is assumed as the reference theory for the investigation of applicability and limitations of the simplified thin elastic shell models.

The starting point of the present paper is represented by the simplified thin elastic shell theory developed by Manevitch for vibration modelling of SWCNTs and reported in Ref. [62]. Specifically, Manevitch proposed a reduced version of Sanders-Koiter shell theory based on the hypotheses of the membrane shell theory (negligible middle surface changes in curvature and torsion) for axisymmetric modes and a combination of the hypotheses of semi-membrane and moment shell theories (negligible middle surface circumferential and shear strains) for beam-like and shell-like modes. Comparisons with the complete (extended) Sanders-Koiter shell theory proved the applicability of reduced (simplified) Sanders-Koiter shell theory for relatively long and thick SWCNTs. Later, Manevitch and Kaplunov [63,64] refined the previously adopted assumptions on the vanishing of circumferential and shear middle surface deformations by means of asymptotic analysis in the vicinity of the lowest cut-off frequency.

In the present paper, the investigation on the applicability and limitations of simplified models of thin elastic shells for SWCNT linear vibrations of Ref. [62] is extended to DWCNTs, where the effect of the van der Waals interaction forces between the adjacent layers completely changes the scenario. The Sanders-Koiter shell theory, retaining three middle surface strains and three middle surface changes in curvature and torsion, is applied as 
reference model. Three different simplified models are compared with Sanders-Koiter shell theory: (i) a shell model retaining only the three middle surface strains, based on the assumptions of membrane shell theory, (ii) a shell model retaining only the three middle surface changes in curvature and torsion, based on the assumptions of moment shell theory, (iii) a shell model retaining the three middle surface strains and the middle surface change in curvature along the circumferential direction, obtained combining membrane and semi-moment shell theories.

The aim of the present study is to propose a new simplified elastic shell model applicable with satisfactory accuracy for linear vibrations of DWCNTs in a large range of geometries and wavenumbers, including cases in which membrane and moment shell models result in large relative errors. This new simplified shell model, as a reduced version of Sanders-Koiter shell theory, by presenting a lower number of middle surface components within the expression of the elastic strain energy, gives rise to a lower computational effort into the numerical simulations with respect to extended Sanders-Koiter shell theory.

\section{Extended Sanders-Koiter Shell Theory}

In the present paper, the actual discrete DWCNT of Figure 1a is modelled by means of a couple of concentric equivalent continuous thin elastic cylindrical shells with van der Waals interaction forces. In Figure $1 b, c$, a continuous thin elastic cylindrical shell with radius $R$, length $L$ and thickness $h$ is shown; a cylindrical coordinate system $(O ; x, \theta, z)$ is considered, where the origin $O$ of the reference system is located at the centre of one end of the cylindrical shell. Three different displacements are present: longitudinal $u(x, \theta, t)$, circumferential $v(x, \theta, t)$ and radial $w(x, \theta, t)$; the radial displacement $w$ is assumed positive outward; $(x, \theta)$ are the longitudinal and angular coordinates of an arbitrary point on the middle surface of the shell; $z$ is the radial coordinate along the thickness $h$; $t$ is the time.

(b)

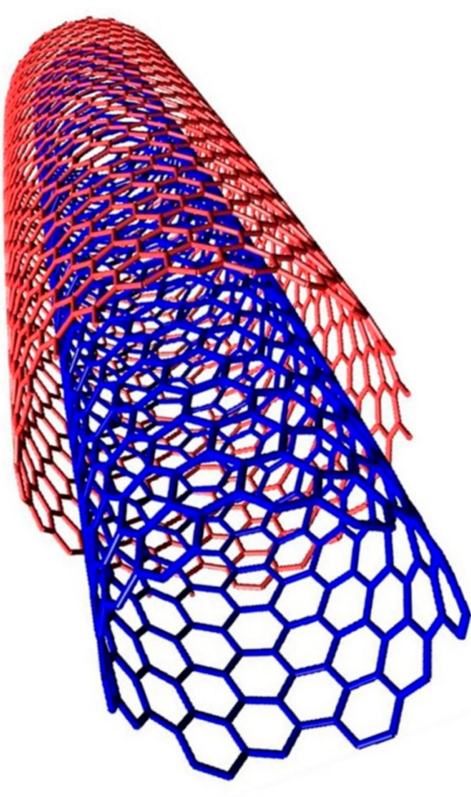

(a)

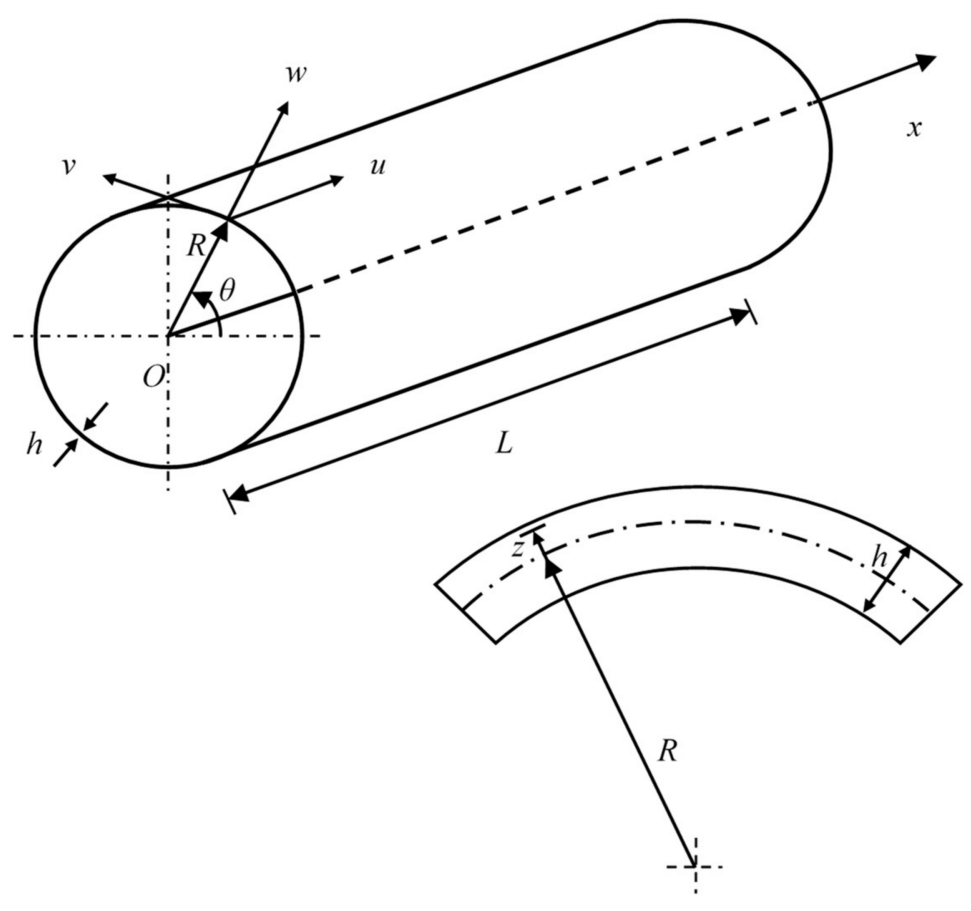

(c)

Figure 1. Continuum modelling of a DWCNT. (a) Actual discrete DWCNT. (b) Geometry of the equivalent continuous single cylindrical shell. (c) Cross-section of the surface of the equivalent continuous single cylindrical shell. 


\subsection{Strain-Displacement Relationships}

The dimensionless displacement field of the $i$-th cylindrical shell is written in the form [61]:

$$
\widetilde{u}_{i}=\frac{u_{i}}{R_{i}} \quad \widetilde{v}_{i}=\frac{v_{i}}{R_{i}} \quad \widetilde{w}_{i}=\frac{w_{i}}{R_{i}} \quad i=1,2
$$

where $\left(u_{i}, v_{i}, w_{i}\right)$ is the dimensional displacement field and $R_{i}$ is the radius of the $i$-th shell.

In this paper, the Sanders-Koiter shell theory is used to model the DWCNT dynamics. The linear relationships between strains and displacements in Sanders-Koiter shell theory are based on "Kirchhoff-Love's assumptions" [7]: (i) the thickness of the shell $h$ is small if compared with the other dimensions of the shell, for example the radius of curvature of the middle surface $R$ and the length $L$, (ii) strains and displacements are sufficiently small so that the quantities of second and higher-order magnitude into the strain-displacement relationships may be neglected in comparison with the first-order terms, (iii) the transverse normal stress is small with respect to the other normal stress components and may be neglected (i.e., $\sigma_{z}=0$ ), (iv) normals to the undeformed middle surface remain straight and normal to the deformed middle surface, suffering no extension ("Kirchhoff's kinematic hypothesis").

As for the first geometric assumption, with regard to the thickness ratio $R / h$, it was demonstrated that the range of validity of thin shell theories, in the present work SandersKoiter thin shell theory, is given by $20 \leq R / h \leq 500$, see Ref. [16] for more details. Moreover, the consequence of the last geometric assumption is that the transverse shear strains of the thin circular cylindrical shell may be neglected $\left(\gamma_{x z}=\gamma_{\theta z}=0\right)$; in addition, also the rotary inertia of the shell is neglected.

According to the Sanders-Koiter shell theory, the dimensionless strain components at an arbitrary point of the $i$-th shell are related to the dimensionless middle surface strains and changes in curvature and torsion of the $i$-th shell by the following relationships [61]:

$$
\widetilde{\varepsilon}_{x, i}=\widetilde{\varepsilon}_{x, 0, i}+\zeta_{i} \widetilde{k}_{x, i} \quad \widetilde{\varepsilon}_{\theta, i}=\widetilde{\varepsilon}_{\theta, 0, i}+\zeta_{i} \widetilde{k}_{\theta, i} \quad \widetilde{\gamma}_{x \theta, i}=\widetilde{\gamma}_{x \theta, 0, i}+\zeta_{i} \widetilde{k}_{x \theta, i} \quad i=1,2
$$

where $\zeta_{i}=z_{i} / R_{i}$ is the dimensionless radial coordinate of the $i$-th cylindrical shell.

The dimensionless middle surface linear strains of the $i$-th cylindrical shell are [61]:

$$
\widetilde{\varepsilon}_{x, 0, i}=\alpha_{i} \frac{\partial \widetilde{u}_{i}}{\partial \eta} \quad \widetilde{\varepsilon}_{\theta, 0, i}=\frac{\partial \widetilde{v}_{i}}{\partial \theta}+\widetilde{w}_{i} \quad \widetilde{\gamma}_{x \theta, 0, i}=\frac{\partial \widetilde{u}_{i}}{\partial \theta}+\alpha_{i} \frac{\partial \widetilde{v}_{i}}{\partial \eta} \quad i=1,2
$$

where $\eta=x / L$ is the dimensionless longitudinal coordinate of the shell and $\alpha_{i}=R_{i} / L$.

The dimensionless middle surface changes in curvature and torsion of the $i$-th shell are [61]:

$$
\widetilde{k}_{x, i}=-\alpha_{i}^{2} \frac{\partial^{2} \widetilde{w}_{i}}{\partial \eta^{2}} \quad \widetilde{k}_{\theta, i}=\frac{\partial \widetilde{v}_{i}}{\partial \theta}-\frac{\partial^{2} \widetilde{w}_{i}}{\partial \theta^{2}} \quad \widetilde{k}_{x \theta, i}=-2 \alpha_{i} \frac{\partial^{2} \widetilde{w}_{i}}{\partial \eta \partial \theta}+\frac{1}{2}\left(3 \alpha_{i} \frac{\partial \widetilde{v}_{i}}{\partial \eta}-\frac{\partial \widetilde{u}_{i}}{\partial \theta}\right) \quad i=1,2
$$

\subsection{Force and Moment Resultants}

The dimensionless force and moment resultants can be written as functions of the dimensionless middle surface strains and changes in curvature and torsion of the $i$-th cylindrical shell [61]:

$$
\begin{array}{lll}
\widetilde{N}_{x, i}=\frac{1}{\left(1-v^{2}\right)}\left(\widetilde{\mathcal{E}}_{x, 0, i}+v \widetilde{\varepsilon}_{\theta, 0, i}\right) & \widetilde{N}_{\theta, i}=\frac{1}{\left(1-v^{2}\right)}\left(\widetilde{\varepsilon}_{\theta, 0, i}+v \widetilde{\mathcal{E}}_{x, 0, i}\right) & \widetilde{N}_{x \theta, i}=\frac{1}{2(1+v)} \widetilde{\gamma}_{x \theta, 0, i} \\
\widetilde{M}_{x, i}=\frac{\beta_{i}}{12\left(1-v^{2}\right)}\left(\widetilde{k}_{x, i}+v \widetilde{k}_{\theta, i}\right) & \widetilde{M}_{\theta, i}=\frac{\beta_{i}}{12\left(1-v^{2}\right)}\left(\widetilde{k}_{\theta, i}+v \widetilde{k}_{x, i}\right) & \widetilde{M}_{x \theta, i}=\frac{\beta_{i}}{24(1+v)} \widetilde{k}_{x \theta, i} \quad i=1,2 \\
\widetilde{Q}_{x, i}=\beta_{i}\left(\alpha_{i} \frac{\partial \widetilde{M}_{x, i}}{\partial \eta}+\frac{\partial \widetilde{M}_{x \theta, i}}{\partial \theta}\right) & \widetilde{Q}_{\theta, i}=\beta_{i}\left(\alpha_{i} \frac{\partial \widetilde{M}_{x \theta, i}}{\partial \eta}+\frac{\partial \widetilde{M}_{\theta, i}}{\partial \theta}\right) &
\end{array}
$$

where $v$ is the Poisson's ratio of the cylindrical shell and $\beta_{i}=h / R_{i}$. 


\subsection{Membrane Approximate Shell Theory}

A thin elastic shell supports an arbitrary external loading by means of stress resultants (internal forces) and stress couples (bending and twisting moments), see dimensionless force and moment resultants (5).

However, under appropriate loading and boundary conditions, the resulting bending and twisting moments are either zero, or so small that they may be neglected [3].

Such a state of stress is referred to as membrane state of stress because of the analogy to membranes that cannot support bending and twisting moments. The corresponding theory of thin shells that deals with this state of stress is called membrane theory of thin shells [3].

It follows from the above that the membrane theory neglects all moment resultants, i.e.,

$$
\widetilde{M}_{x, i}=\widetilde{M}_{\theta, i}=\widetilde{M}_{x \theta, i}=0 \quad i=1,2
$$

From the corresponding moment relations (5), it follows that neglecting the moments can be justified by assuming negligible changes in curvature and torsion of the $i$-th shell middle surface:

$$
\widetilde{k}_{x, i}=\widetilde{k}_{\theta, i}=\widetilde{k}_{x \theta, i}=0 \quad i=1,2
$$

and therefore we deal with the membrane or momentless state of stress of the shell [4].

\subsection{Moment Approximate Shell Theory}

Similarly, under appropriate loading and boundary conditions, the resulting internal forces are either zero, or so small that they may be neglected [3].

Such a state of stress is referred to as moment state of stress, where the corresponding theory of thin shells is called moment theory of thin shells [3].

It follows from the above that the moment theory neglects all force resultants, i.e.,

$$
\widetilde{N}_{x, i}=\widetilde{N}_{\theta, i}=\widetilde{N}_{x \theta, i}=0 \quad i=1,2
$$

From the corresponding force relations (5), it follows that neglecting the forces can be justified by assuming very small, at least negligible strains of the $i$-th shell middle surface:

$$
\widetilde{\varepsilon}_{x, 0, i}=\widetilde{\varepsilon}_{\theta, 0, i}=\widetilde{\gamma}_{x \theta, 0, i}=0 \quad i=1,2
$$

and therefore we deal with the moment or forceless state of stress of the shell [4].

\subsection{Stress-Strain Relationships}

Considering plane stress hypothesis ( $\sigma_{z}=0$, Kirchhoff-Love's assumptions), the dimensionless stresses of the $i$-th thin circular cylindrical shell are related to the corresponding dimensionless strains, in case of homogeneous isotropic elastic material, by the following local constitutive equations [61]:

$$
\widetilde{\sigma}_{x, i}=\frac{1}{\left(1-v^{2}\right)}\left(\widetilde{\varepsilon}_{x, i}+v \widetilde{\varepsilon}_{\theta, i}\right) \quad \widetilde{\sigma}_{\theta, i}=\frac{1}{\left(1-v^{2}\right)}\left(\widetilde{\varepsilon}_{\theta, i}+v \widetilde{\varepsilon}_{x, i}\right) \quad \widetilde{\tau}_{x \theta, i}=\frac{1}{2(1+v)} \widetilde{\gamma}_{x \theta, i} \quad i=1,2
$$

\subsection{Elastic Strain Energy}

The dimensionless elastic strain energy of the $i$-th thin elastic circular cylindrical shell, which models a SWCNT, under plane stress hypothesis $\left(\sigma_{z}=0\right)$, is expressed as [61]:

$$
\widetilde{U}_{i}=\frac{1}{2 \beta_{i}} \int_{0}^{1} \int_{0}^{2 \pi} \int_{-\beta_{i} / 2}^{\beta_{i} / 2}\left(\widetilde{\sigma}_{x, i} \widetilde{\varepsilon}_{x, i}+\widetilde{\sigma}_{\theta, i} \widetilde{\varepsilon}_{\theta, i}+\widetilde{\tau}_{x \theta, i} \widetilde{\gamma}_{x \theta, i}\right) d \eta d \theta d \zeta \quad i=1,2
$$


By inserting Equations (2) and (10) into Equation (11), the following expression for the elastic strain energy of the $i$-th circular cylindrical shell is obtained [61]:

$$
\begin{aligned}
\widetilde{U}_{i}= & \frac{1}{2} \frac{1}{\left(1-v^{2}\right)} \int_{0}^{1} \int_{0}^{2 \pi}\left(\widetilde{\varepsilon}_{x, 0, i}^{2}+\widetilde{\varepsilon}_{\theta, 0, i}^{2}+2 v \widetilde{\varepsilon}_{x, 0, i} \widetilde{\varepsilon}_{\theta, 0, i}+\frac{(1-v)}{2} \widetilde{\gamma}_{x \theta, 0, i}^{2}\right) d \eta d \theta+i=1,2 \\
& +\frac{1}{2} \frac{\beta_{i}^{2}}{12\left(1-v^{2}\right)} \int_{0}^{1} \int_{0}^{2 \pi}\left(\widetilde{k}_{x, i}^{2}+\widetilde{k}_{\theta, i}^{2}+2 v \widetilde{k}_{x, i} \widetilde{k}_{\theta, i}+\frac{(1-v)}{2} \widetilde{k}_{x \theta, i}^{2}\right) d \eta d \theta
\end{aligned}
$$

In Equation (12), the first term on the right-hand side, which is related to shell middle surface strains, is referred to as membrane (i.e., stretching) energy, while the second one, which is related to shell middle surface changes in curvature and torsion, is referred to as bending (i.e., flexural) energy. NTs, is:

The dimensionless elastic strain energy of a DWCNT, given by two concentric SWC-

$$
\widetilde{U}=\sum_{i=1}^{2} \delta_{i} \widetilde{U}_{i}
$$

where $\delta_{i}=R_{i} / R_{1}$ and $R_{1}$ is the radius of the inner shell.

\subsection{Kinetic Energy}

The dimensional time variable $t$ is made dimensionless by adopting a reference frequency $\omega_{0}$, which is the lowest extensional circular frequency of a ring under plane strain hypothesis, in the form [61]:

$$
\omega_{0}=\sqrt{\frac{E}{\rho\left(1-v^{2}\right) R_{1}^{2}}} \tau=\omega_{0} t
$$

where $E$ is the Young's modulus, $v$ is the Poisson's ratio and $\rho$ is the mass density of the two layers of the DWCNT, and $\tau$ is the dimensionless time variable.

The dimensionless velocity field of the $i$-th cylindrical shell is written in the form [61]:

$$
\widetilde{u}_{i}^{\prime}=\frac{d \widetilde{u}_{i}}{d \tau}=\frac{\dot{u}_{i}}{R_{i} \omega_{0}} \quad \widetilde{v}_{i}^{\prime}=\frac{d \widetilde{v}_{i}}{d \tau}=\frac{\dot{v}_{i}}{R_{i} \omega_{0}} \quad \widetilde{w}_{i}^{\prime}=\frac{d \widetilde{w}_{i}}{d \tau}=\frac{\dot{w}_{i}}{R_{i} \omega_{0}} \quad i=1,2
$$

where $\left(\dot{u}_{i}, \dot{v}_{i}, \dot{w}_{i}\right)$ is the corresponding dimensional velocity field.

The dimensionless kinetic energy of the $i$-th thin cylindrical shell, which models a SWCNT, by neglecting the rotary inertia effect, is given by [61]:

$$
\widetilde{T}_{i}=\frac{1}{2} \frac{1}{\left(1-v^{2}\right)} \delta_{i}^{2} \int_{0}^{1} \int_{0}^{2 \pi}\left(\widetilde{u}_{i}^{2}+\widetilde{v}_{i}^{2}+\widetilde{w}_{i}^{2}\right) d \eta d \theta \quad i=1,2
$$

The dimensionless kinetic energy of a DWCNT, given by two concentric SWCNTs, is:

$$
\widetilde{T}=\sum_{i=1}^{2} \delta_{i} \widetilde{T}_{i}
$$

\subsection{Van der Waals Interaction Energy}

The dimensionless pressure exerted on the $i$-th circular cylindrical shell due to van der Waals interactions between the two layers $(i, j)$ of a DWCNT is written as a function of the dimensionless radial displacements $\left(\widetilde{w}_{i}, \widetilde{w}_{j}\right)$ of the two layers in the form [61]:

$$
\widetilde{p}_{i}(\eta, \theta)=\widetilde{c}_{i j}\left(\delta_{i} \widetilde{w}_{i}-\delta_{j} \widetilde{w}_{j}\right) \quad i, j=1,2 \quad i \neq j
$$


where $\widetilde{c}_{i j}$ is the dimensionless van der Waals interaction coefficient between layers $(i, j)$, which can be expressed, by adopting the formulation proposed by He et al. [51], as:

$$
\widetilde{c}_{i j}=-\left(\frac{1001 \pi \widetilde{\varepsilon} \widetilde{\sigma}^{12}}{3 \widetilde{a}^{4}} \widetilde{E}_{i j}^{13}-\frac{1120 \pi \widetilde{\widetilde{\sigma}} \widetilde{G}^{6}}{9 \widetilde{E}^{4}} \widetilde{E}_{i j}^{7}\right) \delta_{j} \quad i, j=1,2 \quad i \neq j
$$

where $(\widetilde{\sigma}, \widetilde{\varepsilon}, \widetilde{a})$ are dimensionless van der Waals interaction parameters, see [61] for more details.

The dimensionless elliptical integrals of Equation (19) are given by [51]:

$$
\widetilde{E}_{i j}^{m}=\left(\delta_{j}+\delta_{i}\right)^{-m} \int_{0}^{\pi / 2} \frac{d \theta}{\left(1-\widetilde{k}_{i j} \cos ^{2} \theta\right)^{m / 2}} \quad i, j=1,2 \quad i \neq j \quad m=7,13
$$

and the dimensionless geometric coefficient of Equation (20) is expressed as [51]:

$$
\widetilde{k}_{i j}=\frac{4 \delta_{j} \delta_{i}}{\left(\delta_{j}+\delta_{i}\right)^{2}} \quad i, j=1,2 \quad i \neq j
$$

The dimensionless van der Waals interaction energy of the $i$-th thin cylindrical shell, which models a SWCNT, is expressed as follows [61]:

$$
\widetilde{V}_{i}=-\frac{1}{2} \frac{1}{\beta_{i}} \int_{0}^{1} \int_{0}^{2 \pi} \widetilde{p}_{i}(\eta, \theta) \widetilde{w}_{i} d \eta d \theta \quad i=1,2
$$

and therefore the dimensionless van der Waals interaction energy of a DWCNT is:

$$
\widetilde{V}=\sum_{i=1}^{2} \delta_{i} \widetilde{V}_{i}
$$

\section{Linear Vibration Modelling of DWCNTs}

A modal vibration, i.e., a synchronous motion, of a DWCNT can be formally written as [61]:

$$
\begin{aligned}
& \widetilde{u}_{i}(\eta, \theta, \tau)=\widetilde{U}_{i}(\eta, \theta) \widetilde{f}_{i}(\tau) \\
& \widetilde{w}_{i}(\eta, \theta, \tau)=\widetilde{W}_{i}(\eta, \theta) \widetilde{f}_{i}(\tau)
\end{aligned} \quad \widetilde{v}_{i}(\eta, \theta, \tau)=\widetilde{V}_{i}(\eta, \theta) \widetilde{f}_{i}(\tau){ }_{i=1,2}
$$

where $\left(\widetilde{U}_{i}, \widetilde{V}_{i}, \widetilde{W}_{i}\right)$ are the three components of the modal shape of the $i$-th circular cylindrical shell and $\widetilde{f}_{i}$ is the corresponding dimensionless time law, which is supposed to be the same for the three dimensionless displacements $\left(\widetilde{u}_{i}, \widetilde{v}_{i}, \widetilde{w}_{i}\right)$ (modal vibration hypothesis).

The modal shape components $\left(\widetilde{U}_{i}, \widetilde{V}_{i}, \widetilde{W}_{i}\right)$ are expanded by means of a double mixed series in terms of $m$-th degree Chebyshev orthogonal polynomials $T_{m}{ }^{*}(\eta)$ along the longitudinal direction $\eta$ and harmonic functions $(\cos n \theta, \sin n \theta)$ along the circumferential direction $\theta$, in the form [61]:

$$
\begin{gathered}
\widetilde{U}_{i}(\eta, \theta)=\sum_{m=0}^{M_{u}} \sum_{n=0}^{N} \widetilde{U}_{i, m, n} T_{m}^{*}(\eta) \cos n \theta \quad \widetilde{V}_{i}(\eta, \theta)=\sum_{m=0}^{M_{v}} \sum_{n=0}^{N} \widetilde{V}_{i, m, n} T_{m}^{*}(\eta) \sin n \theta \quad i=1,2 \\
\widetilde{W}_{i}(\eta, \theta)=\sum_{m=0}^{M_{w}} \sum_{n=0}^{N} \widetilde{W}_{i, m, n} T_{m}^{*}(\eta) \cos n \theta
\end{gathered}
$$

where $T_{m}{ }^{*}=T_{m}(2 \eta-1), m$ denotes the number of longitudinal half-waves, $n$ represents the number of circumferential waves and $\left(\widetilde{U}_{i, m, n}, \widetilde{V}_{i, m, n}, \widetilde{W}_{i, m, n}\right)$ are unknown coefficients, which can be calculated by imposing boundary conditions.

\subsection{Boundary Conditions}

In this paper, the linear vibrations of simply supported and free-free DWCNTs are investigated. 


\subsubsection{Simply Supported DWCNTs}

In case of simply supported DWCNTs, the boundary conditions are written as [61]:

$$
\widetilde{v}_{i}=0 \quad \widetilde{w}_{i}=0 \quad \widetilde{N}_{x, i}=0 \quad \widetilde{M}_{x, i}=0 \quad \eta=0,1 \quad i=1,2
$$

Starting from conditions (26), taking into consideration Equation (5) for dimensionless force and moment resultants and Equation (24) for dimensionless displacements, together with expansions (25) for the corresponding modal shape components, the following relationships are derived:

$$
\begin{array}{rrr}
\widetilde{V}_{i}(\eta, \theta)=\sum_{m=0}^{M_{v}} \sum_{n=0}^{N} \widetilde{V}_{i, m, n} T_{m}^{*}(\eta) \sin n \theta=0 & \eta=0,1 \quad i=1,2 \\
\widetilde{W}_{i}(\eta, \theta)=\sum_{m=0}^{M_{w}} \sum_{n=0}^{N} \widetilde{W}_{i, m, n} T_{m}^{*}(\eta) \cos n \theta=0 & \eta=0,1 \quad i=1,2 \\
\widetilde{U}_{i, \eta}(\eta, \theta)=\sum_{m=0}^{M_{u}} \sum_{n=0}^{N} \widetilde{U}_{i, m, n} T_{m, \eta}^{*}(\eta) \cos n \theta=0 & \eta=0,1 \quad i=1,2 \\
\widetilde{W}_{i, \eta \eta}(\eta, \theta)=\sum_{m=0}^{M_{w}} \sum_{n=0}^{N} \widetilde{W}_{i, m, n} T_{m, \eta \eta}^{*}(\eta) \cos n \theta=0 & \eta=0,1 \quad i=1,2
\end{array}
$$

where $(\cdot)_{, \eta}=\partial(\cdot) / \partial \eta$ and $(\cdot)_{, \eta \eta}=\partial^{2}(\cdot) / \partial \eta^{2}$.

The linear algebraic system given by Equations (27)-(30) can be solved analytically in terms of coefficients $\left(\widetilde{U}_{i, 1, n}, \widetilde{U}_{i, 2, n}, \widetilde{V}_{i, 0, n}, \widetilde{V}_{i, 1, n}, \widetilde{W}_{i, 0, n}, \widetilde{W}_{i, 1, n}, \widetilde{W}_{i, 2, n}, \widetilde{W}_{i, 3, n}\right)$, for $n \in[0, N]$. Therefore, in the case of simply supported DWCNTs, eight different coefficients for each cylindrical shell are obtained.

\subsubsection{Free-Free DWCNTs}

In case of free-free DWCNTs, the boundary conditions are written as [61]:

$$
\widetilde{N}_{x, i}=0 \quad \widetilde{N}_{x \theta, i}+\widetilde{M}_{x \theta, i}=0 \quad \widetilde{Q}_{x, i}+\frac{\partial \widetilde{M}_{x \theta, i}}{\partial \theta}=0 \quad \widetilde{M}_{x, i}=0 \quad \eta=0,1 \quad i=1,2
$$

It can be observed that the boundary conditions (31), applied at the free edges of the DWCNTs, are of "natural type" (forces and moments) and not of "geometric type" (displacements and velocities); since the Rayleigh-Ritz method is used to solve the equations, then only the "geometric type" boundary conditions (see simply supported ones) have to be exactly satisfied, while the "natural type" boundary conditions will be satisfied by the minimization of the total energy of the system.

\subsection{Rayleigh-Ritz Method}

In the case of MWCNTs, the maximum number of variables needed to describe a general vibration mode with $n$ circumferential waves is given by $N_{p}=N_{s} \times\left(M_{u}+M_{v}+M_{w}\right.$ $+3-p)$, where $N_{s}$ is the number of concentric SWCNTs, $M_{u}=M_{v}=M_{w}$ is the maximum number of longitudinal half-waves considered and $p$ is the number of equations needed to satisfy the boundary conditions.

By means of a specific convergence analysis it was obtained that $M_{u}=M_{v}=M_{w}=11$ provides accurate results with relatively reduced computational effort. Therefore, for the DWCNTs $\left(N_{s}=2\right)$, in the case of simply supported edges, it is imposed $p=8$ and it is found $N_{p}=56$, while in the case of free-free edges, it is imposed $p=0$ and it is found $N_{p}=72$.

For a multi-mode analysis including different values of circumferential waves $n$, the number of degrees of freedom of the system is computed by the relation $N_{\max }=N_{p} \times(N+1)$, where $N$ is the maximum number of circumferential waves considered. 
Equations (24) are inserted in the expressions of elastic strain energy (12), kinetic energy (16) and van der Waals interaction energy (22) in order to compute the value of Rayleigh quotient $R(\widetilde{\boldsymbol{q}})$, where $\widetilde{\boldsymbol{q}}$ is a vector containing all the unknown coefficients of expansions (25), which is defined in the form [61]:

$$
\tilde{\boldsymbol{q}}=\left[\begin{array}{c}
\vdots \\
\widetilde{U}_{i, m, n} \\
\widetilde{V}_{i, m, n} \\
\widetilde{W}_{i, m, n} \\
\vdots
\end{array}\right] \quad i=1,2
$$

After imposing stationarity to Rayleigh quotient, the following classic eigenvalue problem in dimensionless form is obtained [61]:

$$
\left(-\widetilde{\omega}^{2} \tilde{\boldsymbol{M}}+\tilde{\boldsymbol{K}}\right) \tilde{\boldsymbol{q}}=0
$$

which provides approximate dimensionless circular frequencies (eigenvalues $\widetilde{\omega}_{j}$ ) and modal shapes (eigenvectors $\widetilde{\boldsymbol{q}}_{j}$ ), with $j=\left(1,2 \ldots, N_{\max }\right)$, where $\widetilde{\boldsymbol{M}}$ and $\widetilde{\boldsymbol{K}}$ denote the dimensionless mass and stiffness matrices, respectively.

The approximate modal shape of the $j$-th mode of the $i$-th thin circular cylindrical shell is given by Equation (25), where the coefficients $\left(\widetilde{U}_{i, m, n}, \widetilde{V}_{i, m, n}, \widetilde{W}_{i, m, n}\right)$ are replaced with $\left(\widetilde{U}_{i, m, n}^{(j)}, \widetilde{V}_{i, m, n}^{(j)}, \widetilde{W}_{i, m, n}^{(j)}\right)$, which are the components of the $j$-th eigenvector $\widetilde{\boldsymbol{q}}$ of Equation (33).

The vector function [61]:

$$
\tilde{\boldsymbol{W}}^{(j)}(\eta, \theta)=\left[\begin{array}{c}
\widetilde{U}_{i}^{(j)}(\eta, \theta) \\
\widetilde{V}_{i}^{(j)}(\eta, \theta) \\
\widetilde{W}_{i}^{(j)}(\eta, \theta)
\end{array}\right] i=1,2
$$

is the approximation of the $j$-th eigenfunction vector of the original problem.

The components of the $j$-th eigenfunction Vector (34) are normalised imposing [61]:

$$
\max \left[\max \left[\widetilde{U}_{i}^{(j)}(\eta, \theta), \widetilde{V}_{i}^{(j)}(\eta, \theta), \widetilde{W}_{i}^{(j)}(\eta, \theta)\right]\right]=1 \quad i=1,2
$$

\section{Numerical Results}

In the present work, natural frequencies of DWCNTs obtained by using simplified (reduced) thin shell models are compared with the results of the extended (complete) Sanders-Koiter shell theory. The mechanical parameters reported in Table 1 are adopted. DWCNTs with different values of aspect ratio $L / R_{2}$ and thickness ratio $R_{2} / h$ are considered. Vibration modes with different numbers of longitudinal half-waves $m$ and circumferential waves $n$ are investigated.

Table 1. Mechanical parameters of the considered DWCNTs [46].

\begin{tabular}{cc}
\hline Young's Modulus $E[\mathrm{TPa}]$ & 5.5 \\
\hline Poisson's ratio $v$ & 0.19 \\
\hline Mass density $\rho\left[\mathrm{kg} / \mathrm{m}^{3}\right]$ & 11,700 \\
\hline Layer thickness $h[\mathrm{~nm}]$ & 0.066 \\
\hline Interlayer distance $d[\mathrm{~nm}]$ & 0.34 \\
\hline
\end{tabular}




\subsection{Experimental Validation of the Extended Sanders-Koiter Shell Theory for SWCNTs}

In this section, the model based on the extended Sanders-Koiter shell theory is validated in the linear field by means of comparisons with experimental data available in the literature in terms of natural frequencies of the radial breathing modes (RBMs).

The RBM is a specific vibrational mode that is often used to identify experimentally the CNTs by resonant Raman spectroscopy (RRS). This mode corresponds to the "vibrational" numbers $(m=0, n=0)$ and it appears only in case of free-free boundary conditions.

The existing experimental data for the CNT vibrations are mainly focused on the RBMs of SWNTs because the spectrum of the nanotubes is quite complex. Moreover, the natural frequency associated with the RBM of a SWNT is inversely proportional to the diameter of, and is independent from, the length of the carbon nanotube [38].

Resonant Raman spectroscopy (RRS) represents a powerful technique to study the quantum properties of electrons and phonons in carbon nanotubes and to determine the atomic structure of an isolated SWNT. The RBM exhibits strong resonant characteristics in the Raman spectra, because it corresponds to the symmetric in-phase motion of all the carbon atoms in the radial direction of the tube. In this configuration all the carbon atoms undergo the same radial displacement [39].

For the RBMs, the radial displacement $\widetilde{w}(\eta, \theta, \tau)$ is spatially uniform, i.e., it is independent of $\eta$ and $\theta(\widetilde{w}=\widetilde{w}(\tau))$. In this special case, the bending stiffness of the SWNTs does not appear, because the radial breathing vibration does not involve the bending deformation and it corresponds to an uniaxial stress state of the graphene sheet [40].

The comparisons reported in Table 2 show that the extended Sanders-Koiter shell theory gives natural frequencies with a good agreement with the experiments in terms of radial breathing modes (RBMs) of the free-free SWCNT under different diameters, where the relative errors are always less than $5 \%$, and it appears a satisfactory accuracy.

Table 2. Natural frequencies of the radial breathing mode $(m=0, n=0)$. Comparisons between resonant Raman spectroscopy and extended Sanders-Koiter shell theory. Free-free SWCNT with mechanical parameters of Table 1.

\begin{tabular}{cccc}
\hline \multicolumn{3}{c}{ Natural Frequency $\left[\mathbf{1 0}^{\mathbf{1 2}} \mathbf{H z}\right]$} & \\
\hline SWCNT Diameter $\boldsymbol{D}$ [nm] & Resonant Raman Spectroscopy [40] & Extended Sanders-Koiter Shell Theory \\
\hline 1.018 & 7.165 & 6.905 & 3.63 \\
1.036 & 7.105 & 6.785 & 4.50 \\
1.053 & 6.865 & 6.669 & 2.85 \\
1.137 & 6.295 & 6.177 & 1.87 \\
1.409 & 5.276 & 5.025 & 4.76 \\
1.416 & 5.216 & 4.964 & 4.83 \\
1.435 & 5.066 & 4.895 & 3.37 \\
1.467 & 4.947 & 4.788 & 3.21 \\
1.492 & 4.917 & 4.711 & 4.19 \\
1.528 & 4.797 & 4.594 & 4.23 \\
1.540 & 4.737 & 4.559 & 3.76 \\
1.564 & 4.677 & 4.494 & 3.91 \\
1.627 & 4.617 & 4.393 & 4.85 \\
& 4.527 & 4.318 & 3.62 \\
\end{tabular}

\subsection{Comparisons between Extended Sanders-Koiter Shell Theory and Simplified Thin Shell Models}

In the numerical simulations performed to compare simplified and extended shell models, the aspect ratio $L / R_{2}$ assumes values from 10 (relatively short DWCNT) to 50 (long DWCNT) and 100 (very long DWCNT), while the thickness ratio $R_{2} / h$ assumes values from 25 (relatively thick DWCNT) to 50 (thin DWCNT) and 250 (very thin DWCNT). 
It must be observed that, since the thickness ratio $R_{2} / h$ considered in the numerical simulations is within the range of validity $20 \leq R / h \leq 500$ of Kirchhoff-Love's assumptions, then the DWCNT can be modelled by means of two concentric thin elastic circular cylindrical shells, and the Sanders-Koiter thin shell theory can be correctly applied, see ref. [7] for more details.

As previously stated, in the present paper, the actual discrete DWCNTs are modelled by means of couples of concentric equivalent continuous thin cylindrical shells with van der Waals interactions.

In the case of cylindrical shells, the modal shapes associated with each natural frequency can be classified as primarily radial (flexural), longitudinal (axial) or circumferential (torsional). It was demonstrated that the lowest natural frequency is usually associated with the modal shape where the transverse (i.e., radial) displacement $w$ is prevalent, while the other two natural frequencies are usually higher by one order of magnitude and they are associated with modal shapes where the displacements in the tangent plane (i.e., longitudinal $u$ and circumferential $v$ ) dominate, see Refs. $[4,7,8]$ for more details.

Moreover, since a DWCNT is constituted by two concentric cylindrical shells (i.e., SWCNTs), then each modal shape of the DWCNT is present twice, once referred to the inner cylindrical shell (with prevalent radius $R_{1}$ ) and once referred to the outer cylindrical shell (with prevalent radius $R_{2}$ ). As reported in literature [61], between the two corresponding natural frequencies, the one associated with the modal shape referred to the inner cylindrical shell (inner radius $R_{1}$ ) is usually lower.

Therefore, in the following numerical simulations, only the natural frequencies corresponding to modal shapes with prevalent radial displacement $w$ and prevalent inner radius $R_{1}$ will be analysed, which, according to the previous observations, represent the fundamental (i.e., lowest) frequencies of DWCNTs.

In this paper, the extended Sanders-Koiter shell theory, which retains three middle surface strains $\left(\widetilde{\varepsilon}_{x, 0, i}, \widetilde{\varepsilon}_{\theta, 0, i}, \widetilde{\gamma}_{x \theta, 0, i}\right)$ and three middle surface changes in curvature and torsion $\left(\widetilde{k}_{x, i}, \widetilde{k}_{\theta, i}, \widetilde{k}_{x \theta, i}\right)$ in the expression of the elastic strain energy (12), is taken as reference model. Three different simplified elastic shell models are compared with the extended Sanders-Koiter shell theory to investigate their applicability and limitations for vibration modelling of DWCNTs in case of axisymmetric $(n=0)$, beam-like $(n=1)$ and shell-like $(n \geq 2)$ modes.

The first simplified elastic shell model studied is a reduced version of the SandersKoiter shell theory developed on the basis of the assumptions of the membrane shell theory (6) and (7), retaining only the three middle surface strains $\left(\widetilde{\varepsilon}_{x, 0, i}, \widetilde{\varepsilon}_{\theta, 0, i}, \widetilde{\gamma}_{x \theta, 0, i}\right)$ and neglecting the three middle surface changes in curvature and torsion $\left(\widetilde{k}_{x, i}, \widetilde{k}_{\theta, i}, \widetilde{k}_{x \theta, i}\right)$.

The second simplified elastic shell model analysed is a reduced version of the SandersKoiter shell theory developed on the basis of the assumptions of the moment shell theory (8) and (9), retaining only the three middle surface changes in curvature and torsion $\left(\widetilde{k}_{x, i}, \widetilde{k}_{\theta, i}, \widetilde{k}_{x \theta, i}\right)$ and neglecting the three middle surface strains $\left(\widetilde{\varepsilon}_{x, 0, i}, \widetilde{\varepsilon}_{\theta, 0, i}, \widetilde{\gamma}_{x \theta, 0, i}\right)$.

It must be stressed that, for shell-like modes, as reported in literature [3], the membrane shell theory is accurate for relatively short and thin shells, when the effect of the middle surface changes in curvature and torsion $\left(\widetilde{k}_{x, i}, \widetilde{k}_{\theta, i}, \widetilde{k}_{x \theta, i}\right)$ is negligible (very small bending/torsional deformations); conversely, the moment shell theory is accurate for relatively long and thick shells, when the effect of the middle surface strains $\left(\widetilde{\varepsilon}_{x, 0, i}, \widetilde{\varepsilon}_{\theta, 0, i}, \widetilde{\gamma}_{x \theta, 0, i}\right)$ is negligible (very small stretching deformations).

Therefore, for shell-like modes, a third simplified elastic shell model is introduced, which is a new approximate model, developed on the basis of a combination of the hypotheses of the membrane and semi-moment theories of thin shells, retaining the middle surface terms $\left(\widetilde{\varepsilon}_{x, 0, i}, \widetilde{\varepsilon}_{\theta, 0, i}, \widetilde{\gamma}_{x \theta, 0, i}, \widetilde{k}_{\theta, i}\right)$ and neglecting the middle surface changes in curvature and torsion $\left(\widetilde{k}_{x, i}, \widetilde{k}_{x \theta, i}\right)$. 


\subsubsection{Axisymmetric Vibration Modes}

In this section, natural frequencies of simply supported DWCNTs, in case of axisymmetric vibration modes $(n=0)$, are obtained. Different numbers of longitudinal half-waves $m$ are considered. Aspect ratios $L / R_{2}=(10,50,100)$ and thickness ratios $R_{2} / h=(25,50,250)$ are investigated. Different elastic shell theories are compared.

In Tables 3-5, comparisons between extended Sanders-Koiter and simplified membrane shell theories are reported. A negligible percentage difference $(\approx 0 \%)$ is obtained for every number of longitudinal half-waves $m$ and every value of aspect $L / R_{2}$ and thickness $R_{2} / h$ ratios.

In Tables 6-8, comparisons between extended Sanders-Koiter and simplified moment shell theories are reported. A very large percentage difference $(\approx 100 \%)$ is obtained for every number of longitudinal half-waves $m$ and every value of aspect $L / R_{2}$ and thickness $R_{2} / h$ ratios.

Therefore, in case of axisymmetric vibration modes, membrane shell theory, which neglects the middle surface changes in curvature and torsion $\left(\widetilde{k}_{x, i}, \widetilde{k}_{\theta, i}, \widetilde{k}_{x \theta, i}\right)$ and retains the middle surface strains $\left(\widetilde{\varepsilon}_{x, 0, i}, \widetilde{\varepsilon}_{\theta, 0, i}, \widetilde{\gamma}_{x \theta, 0, i}\right)$, gives an excellent agreement, with respect to the extended Sanders-Koiter shell theory, and can be adopted to properly compute the natural frequencies of DWCNTs.

These results, obtained for DWCNTs, confirm the applicability of membrane and limitations of moment thin shell theories for the axisymmetric vibration modelling of simply supported thin circular cylindrical shells [4]. In fact, in this case, membrane (stretching) energy is prevalent, while bending (flexural) energy is negligible, in the expression of the elastic strain energy of the cylindrical shell.

Table 3. Simply supported DWCNT with aspect ratio $L / R_{2}=10$ and thickness ratios $R_{2} / h=(25,50,250)$. Axisymmetric modes $(n=0)$. Comparisons between Sanders-Koiter and membrane shell theories.

\begin{tabular}{|c|c|c|c|}
\hline \multicolumn{3}{|c|}{ Natural Frequency $\left[10^{12} \mathrm{~Hz}\right]$} & \multirow{2}{*}{ Difference $\%$} \\
\hline Mode $(m, n)$ & Sanders-Koiter Shell Theory & Membrane Shell Theory & \\
\hline Aspect ratio $L / R_{2}=10$ & Thickness ratio $R_{2} / h=25$ & & \\
\hline$(1,0)$ & 2.34634 & 2.34634 & 0.00 \\
\hline$(2,0)$ & 2.36193 & 2.36191 & 0.00 \\
\hline$(3,0)$ & 2.42883 & 2.42875 & 0.00 \\
\hline$(4,0)$ & 2.21080 & 2.21053 & 0.01 \\
\hline$(5,0)$ & 2.26693 & 2.26615 & 0.03 \\
\hline Aspect ratio $L / R_{2}=10$ & Thickness ratio $R_{2} / h=50$ & & \\
\hline$(1,0)$ & 1.12536 & 1.12536 & 0.00 \\
\hline$(2,0)$ & 1.13416 & 1.13416 & 0.00 \\
\hline$(3,0)$ & 1.17636 & 1.17635 & 0.00 \\
\hline$(4,0)$ & 1.06656 & 1.06652 & 0.00 \\
\hline$(5,0)$ & 1.08789 & 1.08779 & 0.01 \\
\hline Aspect ratio $L / R_{2}=10$ & Thickness ratio $R_{2} / h=250$ & & \\
\hline$(1,0)$ & 0.21566 & 0.21566 & 0.00 \\
\hline$(2,0)$ & 0.21759 & 0.21759 & 0.00 \\
\hline$(3,0)$ & 0.22817 & 0.22817 & 0.00 \\
\hline$(4,0)$ & 0.20556 & 0.20556 & 0.00 \\
\hline$(5,0)$ & 0.20879 & 0.20879 & 0.00 \\
\hline
\end{tabular}


Table 4. Simply supported DWCNT with aspect ratio $L / R_{2}=50$ and thickness ratios $R_{2} / h=(25,50,250)$. Axisymmetric modes $(n=0)$. Comparisons between Sanders-Koiter and membrane shell theories.

\begin{tabular}{|c|c|c|c|}
\hline \multicolumn{3}{|c|}{ Natural Frequency $\left[10^{12} \mathrm{~Hz}\right]$} & \multirow{2}{*}{ Difference $\%$} \\
\hline Mode $(m, n)$ & Sanders-Koiter Shell Theory & Membrane Shell Theory & \\
\hline Aspect ratio $L / R_{2}=50$ & Thickness ratio $R_{2} / h=25$ & & \\
\hline$(1,0)$ & 2.34288 & 2.34288 & 0.00 \\
\hline$(2,0)$ & 2.34328 & 2.34328 & 0.00 \\
\hline$(3,0)$ & 2.34397 & 2.34397 & 0.00 \\
\hline$(4,0)$ & 2.34498 & 2.34498 & 0.00 \\
\hline$(5,0)$ & 2.34636 & 2.34636 & 0.00 \\
\hline Aspect ratio $L / R_{2}=50$ & Thickness ratio $R_{2} / h=50$ & & \\
\hline$(1,0)$ & 1.12347 & 1.12347 & 0.00 \\
\hline$(2,0)$ & 1.12369 & 1.12369 & 0.00 \\
\hline$(3,0)$ & 1.12407 & 1.12407 & 0.00 \\
\hline$(4,0)$ & 1.12462 & 1.12462 & 0.00 \\
\hline$(5,0)$ & 1.12537 & 1.12537 & 0.00 \\
\hline Aspect ratio $L / R_{2}=50$ & Thickness ratio $R_{2} / h=250$ & & \\
\hline$(1,0)$ & 0.21526 & 0.21526 & 0.00 \\
\hline$(2,0)$ & 0.21530 & 0.21530 & 0.00 \\
\hline$(3,0)$ & 0.21538 & 0.21538 & 0.00 \\
\hline$(4,0)$ & 0.21550 & 0.21550 & 0.00 \\
\hline$(5,0)$ & 0.21566 & 0.21566 & 0.00 \\
\hline
\end{tabular}

Table 5. Simply supported DWCNT with aspect ratio $L / R_{2}=100$ and thickness ratios $R_{2} / h=(25,50,250)$. Axisymmetric modes $(n=0)$. Comparisons between Sanders-Koiter and membrane shell theories.

\begin{tabular}{|c|c|c|c|}
\hline \multicolumn{3}{|c|}{ Natural Frequency $\left[10^{12} \mathrm{~Hz}\right]$} & \multirow{2}{*}{ Difference \% } \\
\hline Mode $(m, n)$ & Sanders-Koiter Shell Theory & Membrane Shell Theory & \\
\hline Aspect ratio $L / R_{2}=100$ & Thickness ratio $R_{2} / h=25$ & & \\
\hline$(1,0)$ & 2.34278 & 2.34278 & 0.00 \\
\hline$(2,0)$ & 2.34288 & 2.34288 & 0.00 \\
\hline$(3,0)$ & 2.34304 & 2.34304 & 0.00 \\
\hline$(4,0)$ & 2.34328 & 2.34328 & 0.00 \\
\hline$(5,0)$ & 2.34359 & 2.34359 & 0.00 \\
\hline Aspect ratio $L / R_{2}=100$ & Thickness ratio $R_{2} / h=50$ & & \\
\hline$(1,0)$ & 1.12342 & 1.12342 & 0.00 \\
\hline$(2,0)$ & 1.12347 & 1.12347 & 0.00 \\
\hline$(3,0)$ & 1.12356 & 1.12356 & 0.00 \\
\hline$(4,0)$ & 1.12369 & 2.12369 & 0.00 \\
\hline$(5,0)$ & 1.12386 & 1.12386 & 0.00 \\
\hline Aspect ratio $L / R_{2}=100$ & Thickness ratio $R_{2} / h=250$ & & \\
\hline$(1,0)$ & 0.21525 & 0.21525 & 0.00 \\
\hline$(2,0)$ & 0.21526 & 0.21526 & 0.00 \\
\hline$(3,0)$ & 0.21528 & 0.21528 & 0.00 \\
\hline$(4,0)$ & 0.21530 & 0.21530 & 0.00 \\
\hline$(5,0)$ & 0.21534 & 0.21534 & 0.00 \\
\hline
\end{tabular}


Table 6. Simply supported DWCNT with aspect ratio $L / R_{2}=10$ and thickness ratios $R_{2} / h=(25,50,250)$. Axisymmetric modes $(n=0)$. Comparisons between Sanders-Koiter and moment shell theories.

\begin{tabular}{|c|c|c|c|}
\hline \multicolumn{3}{|c|}{ Natural Frequency $\left[10^{12} \mathrm{~Hz}\right]$} & \multirow{2}{*}{ Difference \% } \\
\hline Mode $(m, n)$ & Sanders-Koiter Shell Theory & Moment Shell Theory & \\
\hline Aspect ratio $L / R_{2}=10$ & Thickness ratio $R_{2} / h=25$ & & \\
\hline$(1,0)$ & 2.34634 & 0.00243 & 99.9 \\
\hline$(2,0)$ & 2.36193 & 0.00971 & 99.6 \\
\hline$(3,0)$ & 2.42883 & 0.02185 & 99.1 \\
\hline$(4,0)$ & 2.21080 & 0.03884 & 98.2 \\
\hline$(5,0)$ & 2.26693 & 0.06105 & 97.3 \\
\hline Aspect ratio $L / R_{2}=10$ & Thickness ratio $R_{2} / h=50$ & & \\
\hline$(1,0)$ & 1.12536 & 0.00061 & 99.9 \\
\hline$(2,0)$ & 1.13416 & 0.00243 & 99.8 \\
\hline$(3,0)$ & 1.17636 & 0.00546 & 99.5 \\
\hline$(4,0)$ & 1.06656 & 0.00971 & 99.1 \\
\hline$(5,0)$ & 1.08789 & 0.01526 & 98.6 \\
\hline Aspect ratio $L / R_{2}=10$ & Thickness ratio $R_{2} / h=250$ & & \\
\hline$(1,0)$ & 0.21566 & 0.00002 & 100 \\
\hline$(2,0)$ & 0.21759 & 0.00010 & 99.9 \\
\hline$(3,0)$ & 0.22817 & 0.00022 & 99.9 \\
\hline$(4,0)$ & 0.20556 & 0.00039 & 99.8 \\
\hline$(5,0)$ & 0.20879 & 0.00061 & 99.7 \\
\hline
\end{tabular}

Table 7. Simply supported DWCNT with aspect ratio $L / R_{2}=50$ and thickness ratios $R_{2} / h=(25,50,250)$. Axisymmetric modes $(n=0)$. Comparisons between Sanders-Koiter and moment shell theories.

\begin{tabular}{|c|c|c|c|}
\hline \multicolumn{3}{|c|}{ Natural Frequency $\left[10^{12} \mathrm{~Hz}\right]$} & \multirow{2}{*}{ Difference \% } \\
\hline Mode $(m, n)$ & Sanders-Koiter Shell Theory & Moment Shell Theory & \\
\hline Aspect ratio $L / R_{2}=50$ & Thickness ratio $R_{2} / h=25$ & & \\
\hline$(1,0)$ & 2.34288 & 0.00010 & 100 \\
\hline$(2,0)$ & 2.34328 & 0.00039 & 100 \\
\hline$(3,0)$ & 2.34397 & 0.00087 & 100 \\
\hline$(4,0)$ & 2.34498 & 0.00155 & 99.9 \\
\hline$(5,0)$ & 2.34636 & 0.00244 & 99.9 \\
\hline Aspect ratio $L / R_{2}=50$ & Thickness ratio $R_{2} / h=50$ & & \\
\hline$(1,0)$ & 1.12347 & 0.00002 & 100 \\
\hline$(2,0)$ & 1.12369 & 0.00010 & 100 \\
\hline$(3,0)$ & 1.12407 & 0.00022 & 100 \\
\hline$(4,0)$ & 1.12462 & 0.00039 & 100 \\
\hline$(5,0)$ & 1.12537 & 0.00061 & 99.9 \\
\hline Aspect ratio $L / R_{2}=50$ & Thickness ratio $R_{2} / h=250$ & & \\
\hline$(1,0)$ & 0.21526 & 0.00000 & 100 \\
\hline$(2,0)$ & 0.21530 & 0.00000 & 100 \\
\hline$(3,0)$ & 0.21538 & 0.00001 & 100 \\
\hline$(4,0)$ & 0.21550 & 0.00001 & 100 \\
\hline$(5,0)$ & 0.21566 & 0.00002 & 100 \\
\hline
\end{tabular}


Table 8. Simply supported DWCNT with aspect ratio $L / R_{2}=100$ and thickness ratios $R_{2} / h=(25,50,250)$. Axisymmetric modes $(n=0)$. Comparisons between Sanders-Koiter and moment shell theories.

\begin{tabular}{|c|c|c|c|}
\hline \multicolumn{3}{|c|}{ Natural Frequency $\left[10^{12} \mathrm{~Hz}\right]$} & \multirow{2}{*}{ Difference \% } \\
\hline Mode $(m, n)$ & Sanders-Koiter Shell Theory & Moment Shell Theory & \\
\hline Aspect ratio $L / R_{2}=100$ & Thickness ratio $R_{2} / h=25$ & & \\
\hline$(1,0)$ & 2.34278 & 0.00002 & 100 \\
\hline$(2,0)$ & 2.34288 & 0.00010 & 100 \\
\hline$(3,0)$ & 2.34304 & 0.00022 & 100 \\
\hline$(4,0)$ & 2.34328 & 0.00039 & 100 \\
\hline$(5,0)$ & 2.34359 & 0.00061 & 100 \\
\hline Aspect ratio $L / R_{2}=100$ & Thickness ratio $R_{2} / h=50$ & & \\
\hline$(1,0)$ & 1.12342 & 0.00001 & 100 \\
\hline$(2,0)$ & 1.12347 & 0.00002 & 100 \\
\hline$(3,0)$ & 1.12356 & 0.00005 & 100 \\
\hline$(4,0)$ & 1.12369 & 0.00010 & 100 \\
\hline$(5,0)$ & 1.12386 & 0.00015 & 100 \\
\hline Aspect ratio $L / R_{2}=100$ & Thickness ratio $R_{2} / h=250$ & & \\
\hline$(1,0)$ & 0.21525 & 0.00000 & 100 \\
\hline$(2,0)$ & 0.21526 & 0.00000 & 100 \\
\hline$(3,0)$ & 0.21528 & 0.00000 & 100 \\
\hline$(4,0)$ & 0.21530 & 0.00000 & 100 \\
\hline$(5,0)$ & 0.21534 & 0.00001 & 100 \\
\hline
\end{tabular}

Moreover, since, from the computations, it was found that, for axisymmetric vibration modes, the middle surface tangential shear strain is equal to zero $\left(\widetilde{\gamma}_{x \theta, 0, i}=0\right)$, then this last component can be neglected. Therefore, a further simplified model, based on the hypotheses of the semi-membrane shell theory $\left(\widetilde{\gamma}_{x \theta, 0, i}=\widetilde{k}_{x, i}=\widetilde{k}_{\theta, i}=\widetilde{k}_{x \theta, i}=0\right)$ and retaining the middle surface strains $\left(\widetilde{\varepsilon}_{x, 0, i}, \widetilde{\varepsilon}_{\theta, 0, i}\right)$ (two terms instead of six) in the expression of the elastic strain energy (12), can be used. This model again provides an excellent agreement, but with a much lower computational effort, than the extended Sanders-Koiter shell theory. These last comparisons are omitted for the sake of brevity.

In particular, from the performed simulations it was found that, for sufficiently long and thin DWCNTs $\left(L / R_{2} \geq 50, R_{2} / h \geq 50\right)$, the contribution of the circumferential normal strain $\widetilde{\varepsilon}_{\theta, 0, i}$ is strongly prevalent with respect to the one of the longitudinal normal strain $\widetilde{\varepsilon}_{x, 0, i}\left(\widetilde{\varepsilon}_{\theta, 0, i} \gg \widetilde{\varepsilon}_{x, 0, i}\right)$.

\subsubsection{Beam-Like Vibration Modes}

In this section, natural frequencies of simply supported DWCNTs in the case of beamlike modes $(n=1)$ are obtained. Different numbers of longitudinal half-waves $m$ are considered. Aspect ratios $L / R_{2}=(10,50,100)$ and thickness ratios $R_{2} / h=(25,50,250)$ are investigated. Different elastic shell theories are compared.

In Tables 9-11, comparisons between extended Sanders-Koiter and simplified membrane shell theories are reported. Again, a negligible percentage difference $(\approx 0 \%)$ is obtained for every number of longitudinal half-waves $m$ and every value of aspect $L / R_{2}$ and thickness $R_{2} / h$ ratios.

In Tables 12-14, comparisons between extended Sanders-Koiter and simplified moment shell theories are reported. Again, a very large percentage difference $(\approx 100 \%)$ is found for every number of longitudinal half-waves $m$ and every value of aspect $L / R_{2}$ and thickness $R_{2} / h$ ratios.

Therefore, also in case of beam-like vibration modes, membrane shell theory, which neglects the middle surface changes in curvature and torsion $\left(\widetilde{k}_{x, i}, \widetilde{k}_{\theta, i}, \widetilde{k}_{x \theta, i}\right)$ and retains the middle surface strains $\left(\widetilde{\varepsilon}_{x, 0, i}, \widetilde{\widetilde{\varepsilon}}_{\theta, 0, i}, \widetilde{\gamma}_{x \theta, 0, i}\right)$, gives an excellent agreement with respect 
to the extended Sanders-Koiter shell theory and can be adopted to properly compute the natural frequencies of DWCNTs.

These results, obtained for DWCNTs, confirm applicability of membrane and limitations of moment thin shell theories also for beam-like vibration modelling of simply supported thin circular cylindrical shells [4]. In fact, also in this case, membrane energy is prevalent, while bending energy is negligible, in the expression of the elastic strain energy of the cylindrical shell.

From the computations it was obtained that, for beam-like vibration modes, all middle surface strains are not negligible. Therefore, a simplified elastic shell model, based on the hypotheses of the membrane shell theory $\left(\widetilde{k}_{x, i}=\widetilde{k}_{\theta, i}=\widetilde{k}_{x \theta, i}=0\right)$ and taking into account all the middle surface strains $\left(\widetilde{\varepsilon}_{x, 0, i}, \widetilde{\varepsilon}_{\theta, 0, i}, \widetilde{\gamma}_{x \theta, 0, i}\right)$ (three terms instead of six) in the expression of the elastic strain energy (12), can be adopted. This model provides an excellent agreement but with a lower computational effort than the extended Sanders-Koiter shell theory.

In particular, from the performed simulations, similarly to the axisymmetric modes, it was found that, for sufficiently long and thin DWCNTs $\left(L / R_{2} \geq 50, R_{2} / h \geq 50\right)$, the contribution of the circumferential normal strain $\widetilde{\varepsilon}_{\theta, 0, i}$ is strongly prevalent with respect to the longitudinal normal $\widetilde{\varepsilon}_{x, 0, i}$ and tangential shear $\widetilde{\gamma}_{x \theta, 0, i}$ strains $\left(\widetilde{\varepsilon}_{\theta, 0, i} \gg\left(\widetilde{\varepsilon}_{x, 0, i}, \widetilde{\gamma}_{x \theta, 0, i}\right)\right)$.

Therefore, from the results reported in the present and in the previous section, it can be derived that membrane shell theory, as a simplified elastic shell model, provides natural frequencies with excellent agreement with respect to the extended Sanders-Koiter shell theory with regard to axisymmetric and beam-like modes. The goal of the next section is to verify if the membrane shell theory can be rightly applied also to shell-like modes or of it is necessary to integrate/complete it with moment shell theory in order to obtain an acceptable agreement with the results of the extended Sanders-Koiter shell theory.

Table 9. Simply supported DWCNT with aspect ratio $L / R_{2}=10$ and thickness ratios $R_{2} / h=(25,50,250)$. Beam-like vibration modes $(n=1)$. Comparisons between Sanders-Koiter and membrane shell theories.

\begin{tabular}{cccc}
\hline \multicolumn{3}{c}{ Natural Frequency $[\mathbf{1 0} \mathbf{1 2} \mathbf{H z}]$} & Difference \% \\
\cline { 1 - 2 } Mode $(\boldsymbol{m}, \boldsymbol{n})$ & Sanders-Koiter Shell Theory & Membrane Shell Theory & 0.01 \\
Aspect ratio $L / R_{2}=10$ & Thickness ratio $R_{2} / h=25$ & 0.12063 & 0.01 \\
$(1,1)$ & 0.12064 & 0.39060 & 0.03 \\
$(2,1)$ & 0.39066 & 0.70040 & 0.05 \\
$(3,1)$ & 0.70059 & 1.00134 & 0.09 \\
$(5,1)$ & 1.00182 & 1.27195 & 0.00 \\
Aspect ratio $L / R_{2}=10$ & 1.27305 & & 0.00 \\
$(1,1)$ & Thickness ratio $R_{2} / h=50$ & 0.01 \\
$(2,1)$ & 0.06251 & 0.06251 & 0.01 \\
$(3,1)$ & 0.20074 & 0.20073 & 0.35707 \\
$(4,1)$ & 0.35710 & 0.50626 & 0.02 \\
$(5,1)$ & 0.50633 & 0.63729 & \\
$(1,1)$ & 0.63743 & & 0.00 \\
$(2,1)$ & Thickness ratio $R_{2} / h=250$ & 0.01290 & 0.00 \\
$(3,1)$ & 0.01290 & 0.04102 & 0.00 \\
$(5,1)$ & 0.04102 & 0.07235 & 0.00 \\
\hline Aspect ratio $L / R_{2}=10$ & 0.07235 & 0.10179 & 0.00 \\
\hline
\end{tabular}


Table 10. Simply supported DWCNT with aspect ratio $L / R_{2}=50$ and thickness ratios $R_{2} / h=(25,50,250)$. Beam-like modes $(n=1)$. Comparisons between Sanders-Koiter and membrane shell theories.

\begin{tabular}{|c|c|c|c|}
\hline \multicolumn{3}{|c|}{ Natural frequency $\left[10^{12} \mathrm{~Hz}\right]$} & \multirow{2}{*}{ Difference \% } \\
\hline Mode $(m, n)$ & Sanders-Koiter Shell Theory & Membrane Shell Theory & \\
\hline Aspect ratio $L / R_{2}=50$ & Thickness ratio $R_{2} / h=25$ & & \\
\hline$(1,1)$ & 0.00531 & 0.00531 & 0.00 \\
\hline$(2,1)$ & 0.02098 & 0.02098 & 0.00 \\
\hline$(3,1)$ & 0.04620 & 0.04620 & 0.00 \\
\hline$(4,1)$ & 0.07985 & 0.07984 & 0.01 \\
\hline$(5,1)$ & 0.12111 & 0.12109 & 0.02 \\
\hline Aspect ratio $L / R_{2}=50$ & Thickness ratio $R_{2} / h=50$ & & \\
\hline$(1,1)$ & 0.00277 & 0.00277 & 0.00 \\
\hline$(2,1)$ & 0.01091 & 0.01091 & 0.00 \\
\hline$(3,1)$ & 0.02401 & 0.02401 & 0.00 \\
\hline$(4,1)$ & 0.04144 & 0.04144 & 0.00 \\
\hline$(5,1)$ & 0.06275 & 0.06274 & 0.02 \\
\hline Aspect ratio $L / R_{2}=50$ & Thickness ratio $R_{2} / h=250$ & & \\
\hline$(1,1)$ & 0.00057 & 0.00057 & 0.00 \\
\hline$(2,1)$ & 0.00226 & 0.00226 & 0.00 \\
\hline$(3,1)$ & 0.00498 & 0.00498 & 0.00 \\
\hline$(4,1)$ & 0.00857 & 0.00857 & 0.00 \\
\hline$(5,1)$ & 0.01295 & 0.01295 & 0.00 \\
\hline
\end{tabular}

Table 11. Simply supported DWCNT with aspect ratio $L / R_{2}=100$ and thickness ratios $R_{2} / h=(25,50,250)$. Beam-like modes $(n=1)$. Comparisons between Sanders-Koiter and membrane shell theories.

\begin{tabular}{|c|c|c|c|}
\hline \multicolumn{3}{|c|}{ Natural Frequency $\left[10^{12} \mathrm{~Hz}\right]$} & \multirow{2}{*}{ Difference \% } \\
\hline Mode $(m, n)$ & Sanders-Koiter Shell Theory & Membrane Shell Theory & \\
\hline Aspect ratio $L / R_{2}=100$ & Thickness ratio $R_{2} / h=25$ & & \\
\hline$(1,1)$ & 0.00133 & 0.00133 & 0.00 \\
\hline$(2,1)$ & 0.00531 & 0.00531 & 0.00 \\
\hline$(3,1)$ & 0.01189 & 0.01189 & 0.00 \\
\hline$(4,1)$ & 0.02098 & 0.02098 & 0.00 \\
\hline$(5,1)$ & 0.03263 & 0.03263 & 0.00 \\
\hline Aspect ratio $L / R_{2}=100$ & Thickness ratio $R_{2} / h=50$ & & \\
\hline$(1,1)$ & 0.00069 & 0.00069 & 0.00 \\
\hline$(2,1)$ & 0.00277 & 0.00277 & 0.00 \\
\hline$(3,1)$ & 0.00619 & 0.00619 & 0.00 \\
\hline$(4,1)$ & 0.01091 & 0.01091 & 0.00 \\
\hline$(5,1)$ & 0.01697 & 0.01697 & 0.00 \\
\hline Aspect ratio $L / R_{2}=100$ & Thickness ratio $R_{2} / h=250$ & & \\
\hline$(1,1)$ & 0.00014 & 0.00014 & 0.00 \\
\hline$(2,1)$ & 0.00057 & 0.00057 & 0.00 \\
\hline$(3,1)$ & 0.00128 & 0.00128 & 0.00 \\
\hline$(4,1)$ & 0.00226 & 0.00226 & 0.00 \\
\hline$(5,1)$ & 0.00352 & 0.00352 & 0.00 \\
\hline
\end{tabular}


Table 12. Simply supported DWCNT with aspect ratio $L / R_{2}=10$ and thickness ratios $R_{2} / h=(25,50,250)$. Beam-like modes $(n=1)$. Comparisons between Sanders-Koiter and moment shell theories.

\begin{tabular}{|c|c|c|c|}
\hline \multicolumn{3}{|c|}{ Natural Frequency $\left[10^{12} \mathrm{~Hz}\right]$} & \multirow{2}{*}{ Difference \% } \\
\hline Mode $(m, n)$ & Sanders-Koiter Shell Theory & Moment Shell Theory & \\
\hline Aspect ratio $L / R_{2}=10$ & Thickness ratio $R_{2} / h=25$ & & \\
\hline$(1,1)$ & 0.12064 & 0.05079 & 57.9 \\
\hline$(2,1)$ & 0.39066 & 0.05734 & 85.3 \\
\hline$(3,1)$ & 0.70059 & 0.06802 & 90.3 \\
\hline$(4,1)$ & 1.00182 & 0.08297 & 91.7 \\
\hline$(5,1)$ & 1.27305 & 0.10238 & 92.0 \\
\hline Aspect ratio $L / R_{2}=10$ & Thickness ratio $R_{2} / h=50$ & & \\
\hline$(1,1)$ & 0.06251 & 0.01057 & 83.1 \\
\hline$(2,1)$ & 0.20074 & 0.01232 & 93.9 \\
\hline$(3,1)$ & 0.35710 & 0.01512 & 95.8 \\
\hline$(4,1)$ & 0.50633 & 0.01899 & 96.2 \\
\hline$(5,1)$ & 0.63743 & 0.02407 & 96.2 \\
\hline Aspect ratio $L / R_{2}=10$ & Thickness ratio $R_{2} / h=250$ & & \\
\hline$(1,1)$ & 0.01290 & 0.00038 & 97.0 \\
\hline$(2,1)$ & 0.04102 & 0.00045 & 98.9 \\
\hline$(3,1)$ & 0.07235 & 0.00057 & 99.2 \\
\hline$(4,1)$ & 0.10179 & 0.00072 & 99.3 \\
\hline$(5,1)$ & 0.12716 & 0.00093 & 99.3 \\
\hline
\end{tabular}

Table 13. Simply supported DWCNT with aspect ratio $L / R_{2}=50$ and thickness ratios $R_{2} / h=(25,50,250)$. Beam-like modes $(n=1)$. Comparisons between Sanders-Koiter and moment shell theories.

\begin{tabular}{|c|c|c|c|}
\hline \multicolumn{3}{|c|}{ Natural Frequency $\left[10^{12} \mathrm{~Hz}\right]$} & \multirow{2}{*}{ Difference \% } \\
\hline Mode $(m, n)$ & Sanders-Koiter Shell Theory & Moment Shell Theory & \\
\hline Aspect ratio $L / R_{2}=50$ & Thickness ratio $R_{2} / h=25$ & & \\
\hline$(1,1)$ & 0.00531 & 0.00007 & 98.7 \\
\hline$(2,1)$ & 0.02098 & 0.00027 & 98.7 \\
\hline$(3,1)$ & 0.04620 & 0.00060 & 98.7 \\
\hline$(4,1)$ & 0.07985 & 0.00106 & 98.7 \\
\hline$(5,1)$ & 0.12111 & 0.00165 & 98.6 \\
\hline Aspect ratio $L / R_{2}=50$ & Thickness ratio $R_{2} / h=50$ & & \\
\hline$(1,1)$ & 0.00277 & 0.00002 & 99.4 \\
\hline$(2,1)$ & 0.01092 & 0.00007 & 99.4 \\
\hline$(3,1)$ & 0.02401 & 0.00015 & 99.4 \\
\hline$(4,1)$ & 0.04144 & 0.00026 & 99.4 \\
\hline$(5,1)$ & 0.06275 & 0.00041 & 99.3 \\
\hline Aspect ratio $L / R_{2}=50$ & Thickness ratio $R_{2} / h=250$ & & \\
\hline$(1,1)$ & 0.00057 & 0.00000 & 100 \\
\hline$(2,1)$ & 0.00226 & 0.00000 & 100 \\
\hline$(3,1)$ & 0.00498 & 0.00001 & 99.9 \\
\hline$(4,1)$ & 0.00857 & 0.00001 & 99.9 \\
\hline$(5,1)$ & 0.01295 & 0.00002 & 99.9 \\
\hline
\end{tabular}


Table 14. Simply supported DWCNT with aspect ratio $L / R_{2}=100$ and thickness ratios $R_{2} / h=(25,50,250)$. Beam-like modes $(n=1)$. Comparisons between Sanders-Koiter and moment shell theories.

\begin{tabular}{|c|c|c|c|}
\hline \multicolumn{3}{|c|}{ Natural Frequency $\left[10^{12} \mathrm{~Hz}\right]$} & \multirow{2}{*}{ Difference $\%$} \\
\hline Mode $(m, n)$ & Sanders-Koiter Shell Theory & Moment Shell Theory & \\
\hline Aspect ratio $L / R_{2}=100$ & Thickness ratio $R_{2} / h=25$ & & \\
\hline$(1,1)$ & 0.00133 & 0.00002 & 98.7 \\
\hline$(2,1)$ & 0.00531 & 0.00007 & 98.7 \\
\hline$(3,1)$ & 0.01189 & 0.00015 & 98.7 \\
\hline$(4,1)$ & 0.02098 & 0.00027 & 98.7 \\
\hline$(5,1)$ & 0.03263 & 0.00042 & 98.7 \\
\hline Aspect ratio $L / R_{2}=100$ & Thickness ratio $R_{2} / h=50$ & & \\
\hline$(1,1)$ & 0.00069 & 0.00000 & 100 \\
\hline$(2,1)$ & 0.00277 & 0.00002 & 99.4 \\
\hline$(3,1)$ & 0.00619 & 0.00004 & 99.4 \\
\hline$(4,1)$ & 0.01091 & 0.00007 & 99.4 \\
\hline$(5,1)$ & 0.01697 & 0.00010 & 99.4 \\
\hline Aspect ratio $L / R_{2}=100$ & Thickness ratio $R_{2} / h=250$ & & \\
\hline$(1,1)$ & 0.00014 & 0.00000 & 100 \\
\hline$(2,1)$ & 0.00057 & 0.00000 & 100 \\
\hline$(3,1)$ & 0.00128 & 0.00000 & 100 \\
\hline$(4,1)$ & 0.00226 & 0.00000 & 100 \\
\hline$(5,1)$ & 0.00352 & 0.00000 & 100 \\
\hline
\end{tabular}

\subsubsection{Shell-Like Vibration Modes}

In this section, natural frequencies of simply supported DWCNTs in the case of shelllike vibration modes $(n \geq 2)$ are obtained. Different numbers of longitudinal half-waves $m$ are considered. Aspect ratios $L / R_{2}=(10,50,100)$ and thickness ratios $R_{2} / h=(25,50,250)$ are investigated. Different elastic shell theories are compared.

In Figures 2-6, comparisons between extended Sanders-Koiter and simplified membrane shell theories are reported. From these comparisons, the following observations can be made:

- $\quad$ by increasing the number of longitudinal half-waves $m$ the percentage difference decreases (the contribution of the middle surface changes $\left(\widetilde{k}_{x, i}, \widetilde{k}_{\theta, i}, \widetilde{k}_{x \theta, i}\right)$ decreases);

- $\quad$ by increasing the number of circumferential waves $n$ the percentage difference increases (the contribution of the middle surface changes $\left(\widetilde{k}_{x, i}, \widetilde{k}_{\theta, i}, \widetilde{k}_{x \theta, i}\right)$ increases);

- by increasing the aspect ratio $L / R_{2}$ the percentage difference increases (the contribution of the middle surface changes $\left(\widetilde{k}_{x, i}, \widetilde{k}_{\theta, i}, \widetilde{k}_{x \theta, i}\right)$ increases);

- by increasing the thickness ratio $R_{2} / h$ the percentage difference decreases (the contribution of the middle surface changes $\left(\widetilde{k}_{x, i}, \widetilde{k}_{\theta, i}, \widetilde{k}_{x \theta, i}\right)$ decreases). 


\section{diff. \% Sanders-Koiter vs. membrane shell theory $-n=2$}

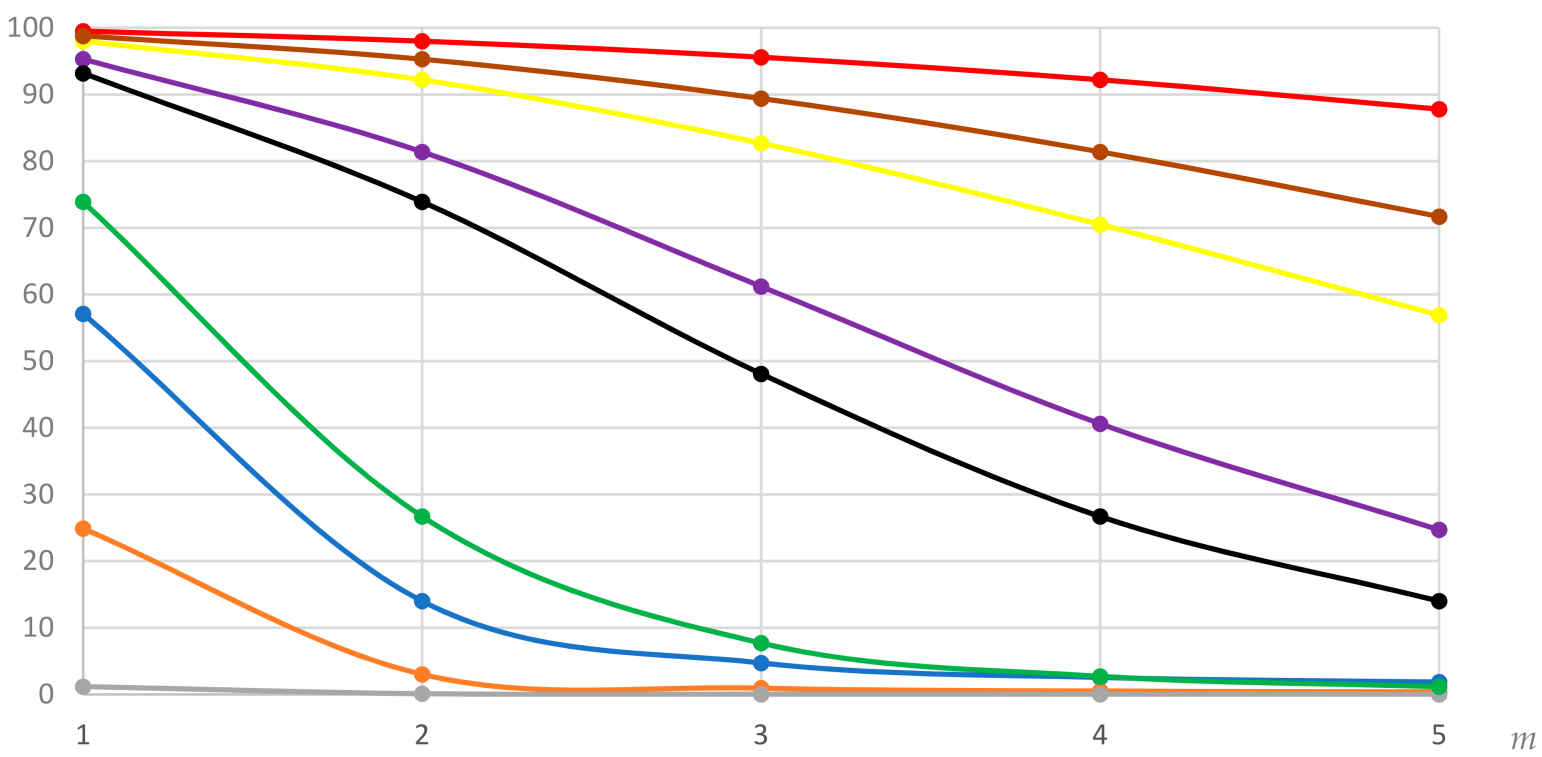

Figure 2. Simply supported DWCNT of Table 1 with different values of aspect ratio $L / R_{2}$ and thickness ratio $R_{2} / h$. Shelllike modes $(n=2)$. Comparisons between extended Sanders-Koiter and simplified membrane shell theories, where $L / R_{2}=10, R_{2} / h=25 ; \quad L / R_{2}=10, R_{2} / h=50 ; \quad L / R_{2}=10, R_{2} / h=250 ; \quad L / R_{2}=50, R_{2} / h=25 ; \quad L / R_{2}=50$, $R_{2} / h=50 ; \quad L / R_{2}=50, R_{2} / h=250 ; \quad L / R_{2}=100, R_{2} / h=25 ; \quad L / R_{2}=100, R_{2} / h=50 ; \quad L / R_{2}=100, R_{2} / h=250$.

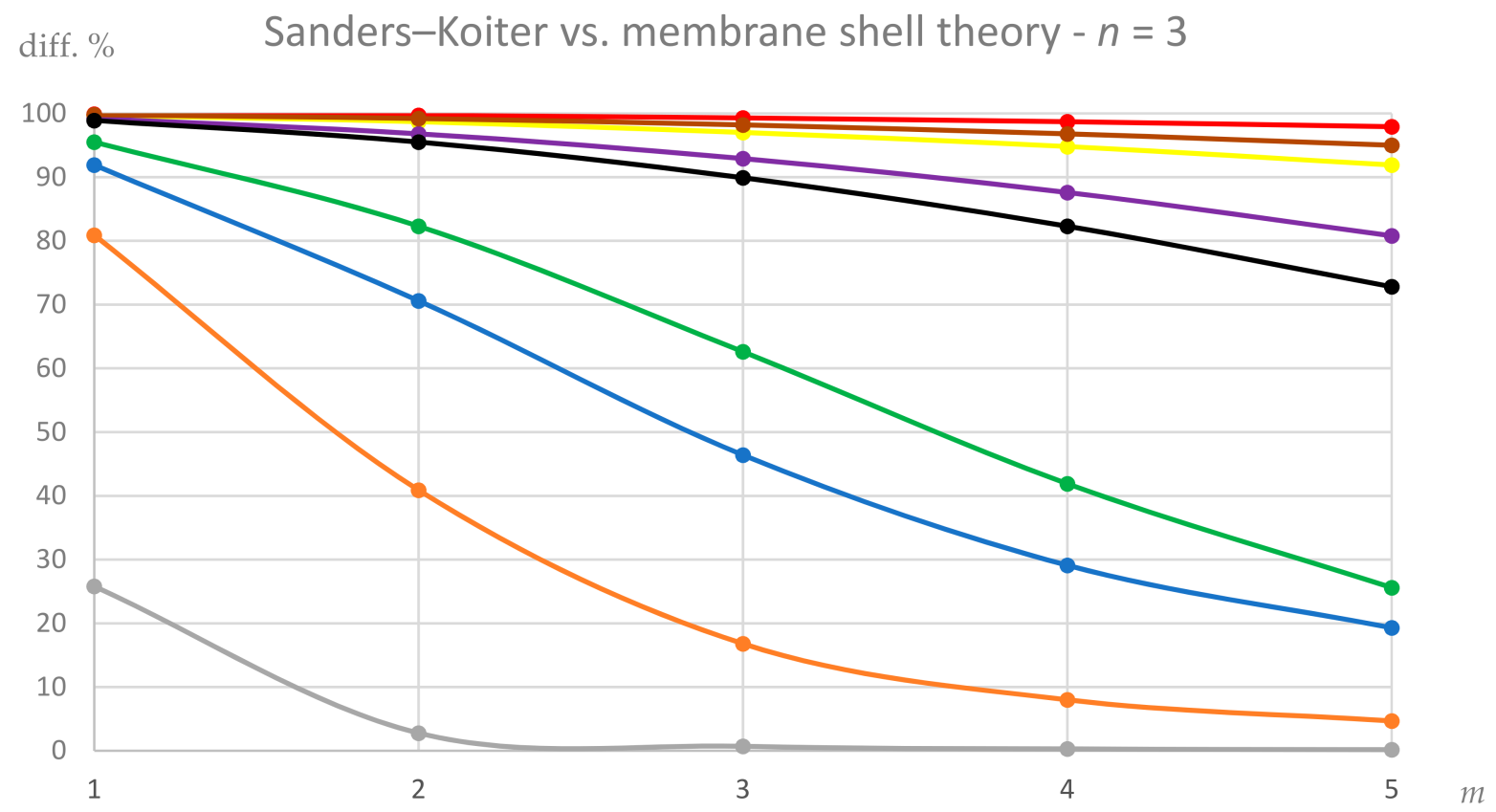

Figure 3. Simply supported DWCNT of Table 1 with different values of aspect ratio $L / R_{2}$ and thickness ratio $R_{2} / h$. Shelllike modes $(n=3)$. Comparisons between extended Sanders-Koiter and simplified membrane shell theories, where $L / R_{2}=10, R_{2} / h=25 ; \quad L / R_{2}=10, R_{2} / h=50 ; \quad L / R_{2}=10, R_{2} / h=250 ; \quad L / R_{2}=50, R_{2} / h=25 ; \quad L / R_{2}=50$ ， $R_{2} / h=50 ; \square L / R_{2}=50, R_{2} / h=250 ; \square L / R_{2}=100, R_{2} / h=25 ; \square L / R_{2}=100, R_{2} / h=50 ; \quad L / R_{2}=100, R_{2} / h=250$. 


\section{diff. $\% \quad$ Sanders-Koiter vs. membrane shell theory $-n=4$}

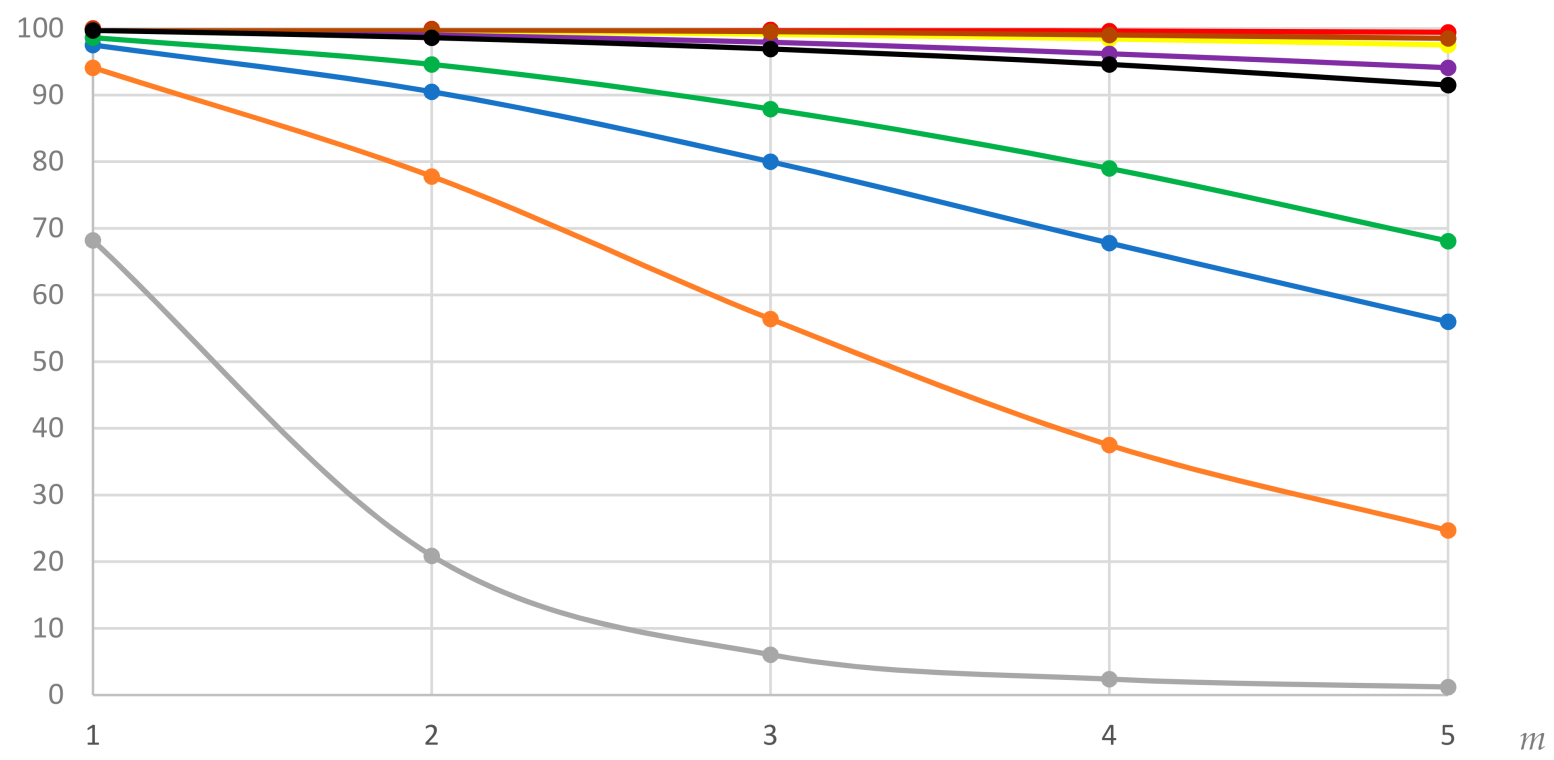

Figure 4. Simply supported DWCNT of Table 1 with different values of aspect ratio $L / R_{2}$ and thickness ratio $R_{2} / h$. Shelllike modes $(n=4)$. Comparisons between extended Sanders-Koiter and simplified membrane shell theories, where $L / R_{2}=10, R_{2} / h=25 ; \quad L / R_{2}=10, R_{2} / h=50 ; \quad L / R_{2}=10, R_{2} / h=250 ; \quad L / R_{2}=50, R_{2} / h=25 ; \quad L / R_{2}=50$, $R_{2} / h=50 ; \quad L / R_{2}=50, R_{2} / h=250 ; \quad L / R_{2}=100, R_{2} / h=25 ; \quad L / R_{2}=100, R_{2} / h=50 ; \quad L / R_{2}=100, R_{2} / h=250$.

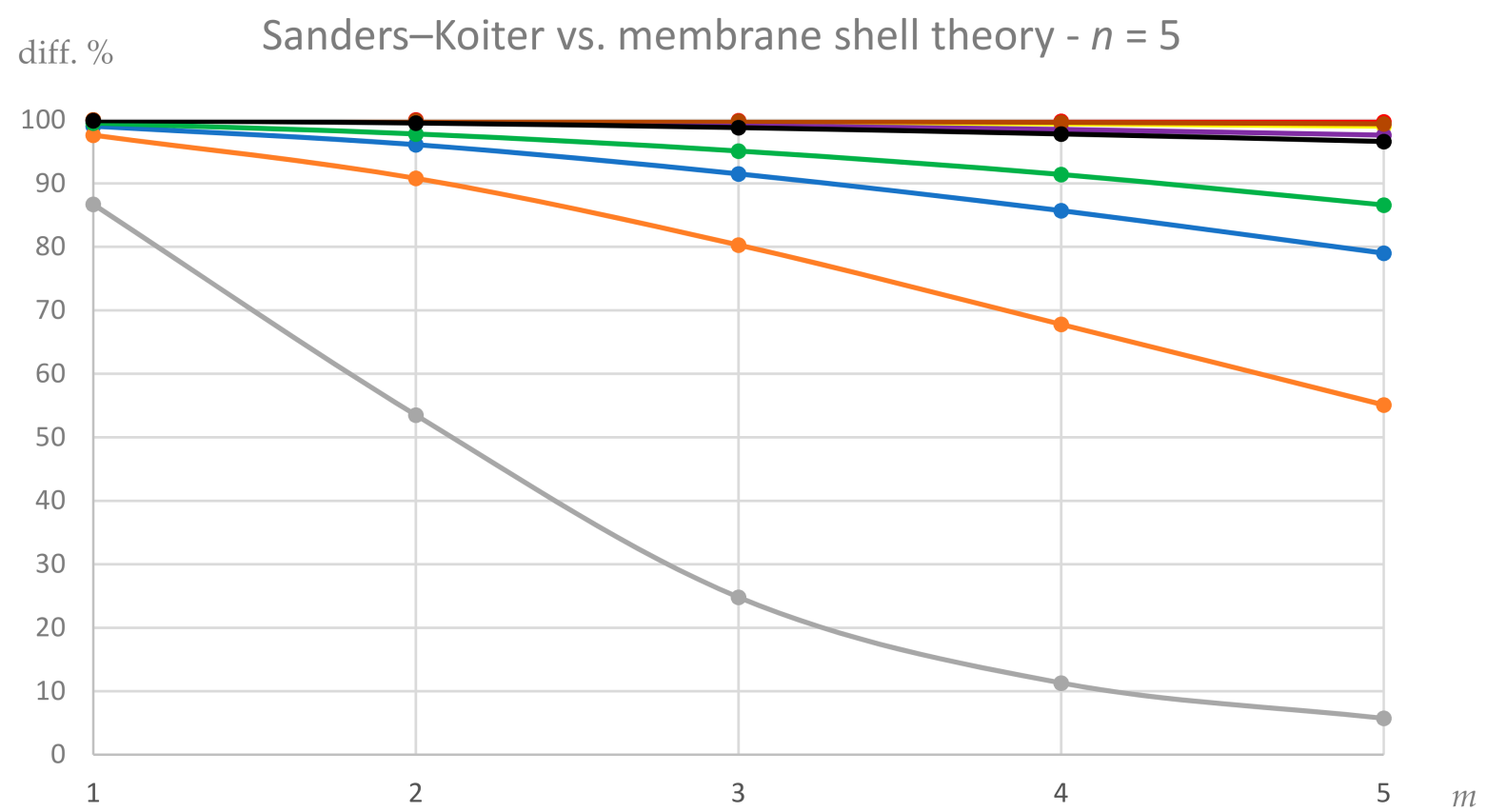

Figure 5. Simply supported DWCNT of Table 1 with different values of aspect ratio $L / R_{2}$ and thickness ratio $R_{2} / h$. Shelllike modes $(n=5)$. Comparisons between extended Sanders-Koiter and simplified membrane shell theories, where $L / R_{2}=10, R_{2} / h=25 ; \square L / R_{2}=10, R_{2} / h=50 ; \quad L / R_{2}=10, R_{2} / h=250 ; \quad L / R_{2}=50, R_{2} / h=25 ; \quad L / R_{2}=50$ ， $R_{2} / h=50 ; \quad L / R_{2}=50, R_{2} / h=250 ; \quad L / R_{2}=100, R_{2} / h=25 ; \quad L / R_{2}=100, R_{2} / h=50 ; \quad L / R_{2}=100, R_{2} / h=250$. 


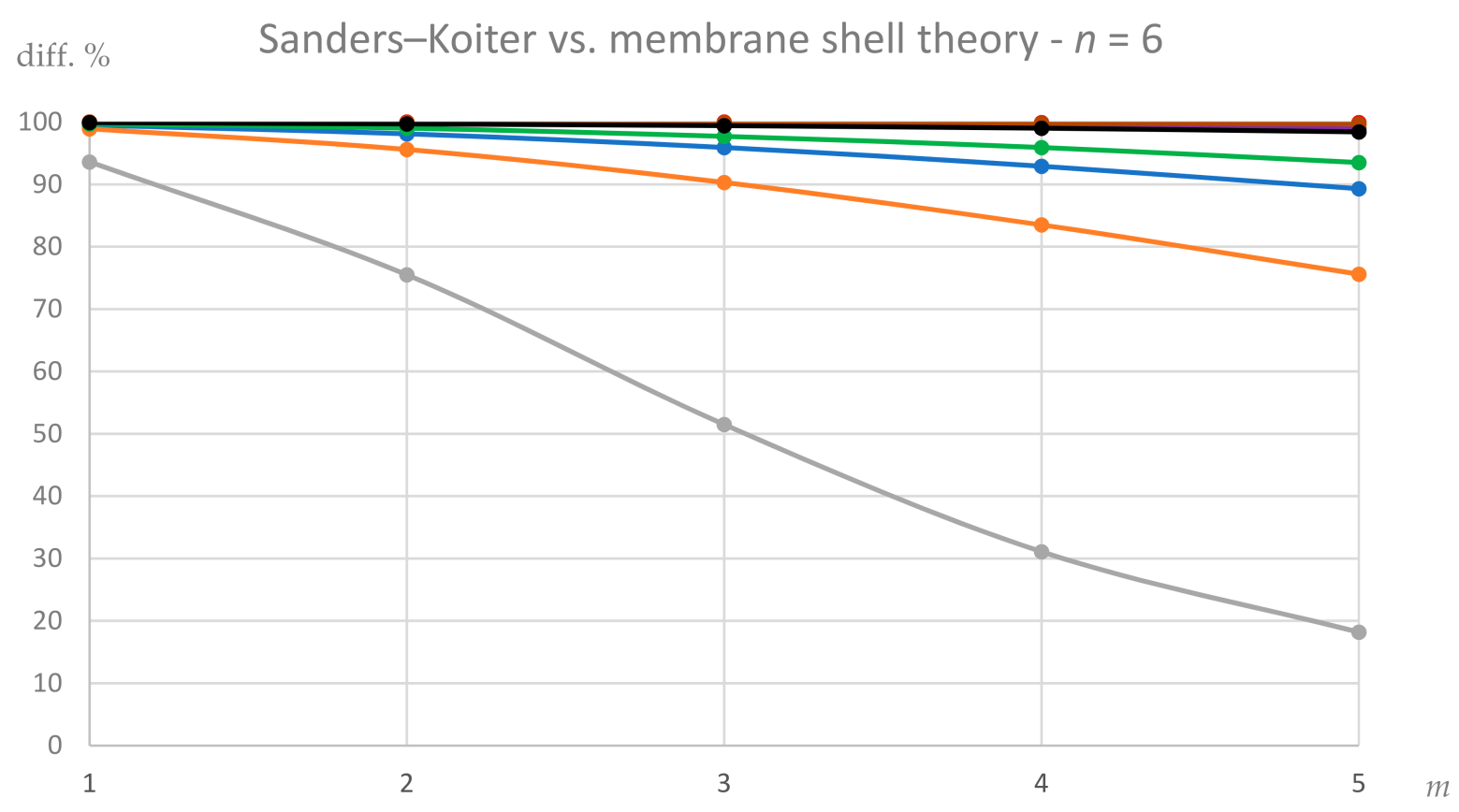

Figure 6. Simply supported DWCNT of Table 1 with different values of aspect ratio $L / R_{2}$ and thickness ratio $R_{2} / h$. Shelllike modes $(n=6)$. Comparisons between extended Sanders-Koiter and simplified membrane shell theories, where $L / R_{2}=10, R_{2} / h=25 ; \quad L / R_{2}=10, R_{2} / h=50 ; \quad L / R_{2}=10, R_{2} / h=250 ; \quad L / R_{2}=50, R_{2} / h=25 ; \quad L / R_{2}=50$, $R_{2} / h=50 ; \quad L / R_{2}=50, R_{2} / h=250 ; \quad L / R_{2}=100, R_{2} / h=25 ; \quad L / R_{2}=100, R_{2} / h=50 ; \quad L / R_{2}=100, R_{2} / h=250$.

Therefore, in case of shell-like modes, membrane shell theory yields satisfactory results only for relatively short and very thin DWCNTs, when the effect of middle surface changes in curvature and torsion $\left(\widetilde{k}_{x, i}, \widetilde{k}_{\theta, i}, \widetilde{k}_{x \theta, i}\right)$ is negligible (very small bending and torsional deformations) and the effect of middle surface strains $\left(\widetilde{\varepsilon}_{x, 0, i}, \widetilde{\varepsilon}_{\theta, 0, i}, \widetilde{\gamma}_{x \theta, 0, i}\right)$ results prevalent (very high stretching deformations).

Starting from the previous observations, the following results can be obtained:

- $\quad$ the percentage difference decreases as the number of longitudinal half-waves $m$ increases, the number of circumferential waves $n$ decreases, the aspect ratio $L / R_{2}$ decreases and the thickness ratio $R_{2} / h$ increases;

- the minimum percentage difference is obtained at $\left(m=5, n=2, L / R_{2}=10, R_{2} / h=250\right)$ and is equal to 0.01 ;

- the percentage difference increases as the number of longitudinal half-waves $m$ decreases, the number of circumferential waves $n$ increases, the aspect ratio $L / R_{2}$ increases and the thickness ratio $R_{2} / h$ decreases;

- the maximum percentage difference is obtained at $\left(m=1, n=6, L / R_{2}=100, R_{2} / h=25\right)$ and is equal to 100 .

In Figures 7-11, comparisons between extended Sanders-Koiter and simplified moment shell theories are reported. From these comparisons, the following observations can be made:

- by increasing the aspect ratio $L / R_{2}$ the percentage difference decreases (the contribution of the middle surface strains $\left(\widetilde{\varepsilon}_{x, 0, i}, \widetilde{\varepsilon}_{\theta, 0, i}, \widetilde{\gamma}_{x \theta, 0, i}\right)$ decreases);

- by increasing the thickness ratio $R_{2} / h$ the percentage difference increases (the contribution of middle surface strains $\left(\widetilde{\varepsilon}_{x, 0, i}, \widetilde{\varepsilon}_{\theta, 0, i}, \widetilde{\gamma}_{x \theta, 0, i}\right)$ increases);

- $\quad$ the curve showing maximum percentage difference corresponds to $\left(L / R_{2}=10, R_{2} / h=250\right)$;

- the curve showing minimum percentage difference corresponds to $\left(L / R_{2}=100\right.$, $\left.R_{2} / h=25\right)$. 


\section{diff. $\% \quad$ Sanders-Koiter vs. moment shell theory $-n=2$}

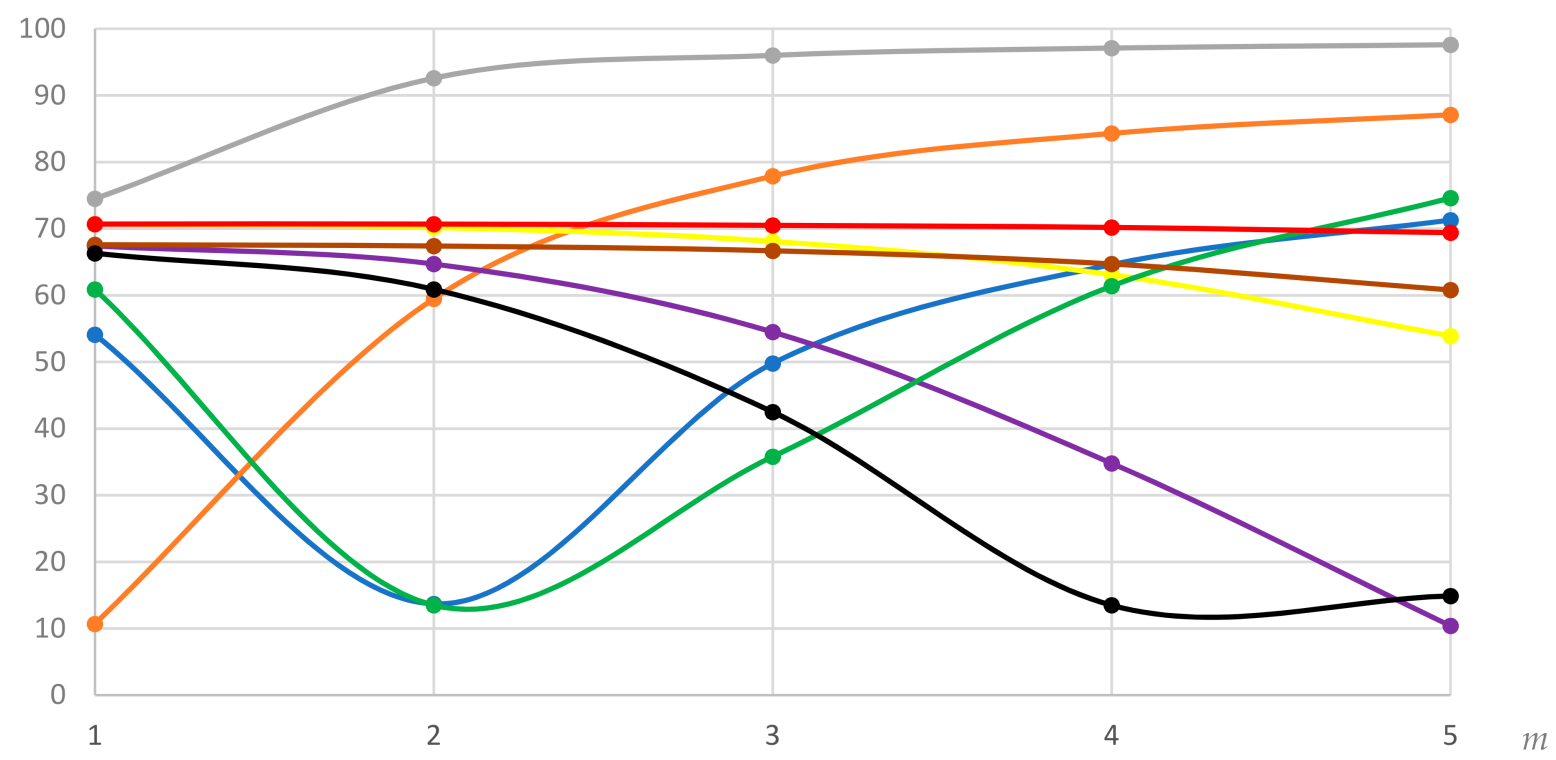

Figure 7. Simply supported DWCNT of Table 1 with different values of aspect ratio $L / R_{2}$ and thickness ratio $R_{2} / h$. Shell-like modes $(n=2)$. Comparisons between extended Sanders-Koiter and simplified moment shell theories, where $L / R_{2}=10$, $R_{2} / h=25 ; \quad L / R_{2}=10, R_{2} / h=50 ; \quad L / R_{2}=10, R_{2} / h=250 ; \quad L / R_{2}=50, R_{2} / h=25 ; \quad L / R_{2}=50, R_{2} / h=50 ;$ $L / R_{2}=50, R_{2} / h=250 ; \quad L / R_{2}=100, R_{2} / h=25 ; \quad L / R_{2}=100, R_{2} / h=50 ; \square L / R_{2}=100, R_{2} / h=250$.

\section{diff. \% Sanders-Koiter vs. moment shell theory $-n=3$}

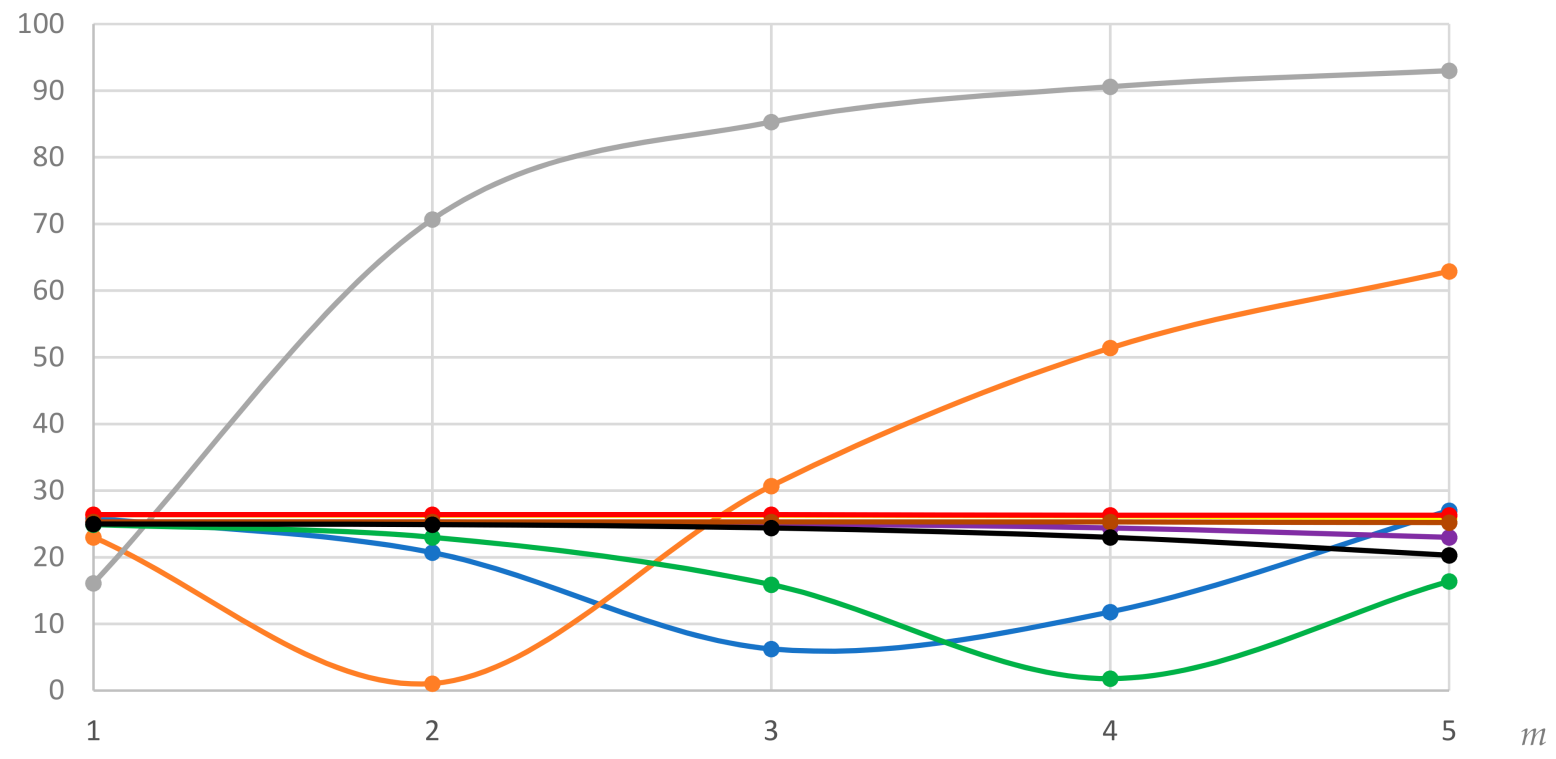

Figure 8. Simply supported DWCNT of Table 1 with different values of aspect ratio $L / R_{2}$ and thickness ratio $R_{2} / h$. Shell-like modes $(n=3)$. Comparisons between extended Sanders-Koiter and simplified moment shell theories, where $L / R_{2}=10$, $R_{2} / h=25 ; \quad L / R_{2}=10, R_{2} / h=50 ; \quad L / R_{2}=10, R_{2} / h=250 ; \quad L / R_{2}=50, R_{2} / h=25 ; \quad L / R_{2}=50, R_{2} / h=50 ;$ $L / R_{2}=50, R_{2} / h=250 ; \quad L / R_{2}=100, R_{2} / h=25 ; \quad L / R_{2}=100, R_{2} / h=50 ; \boldsymbol{L} L / R_{2}=100, R_{2} / h=250$. 


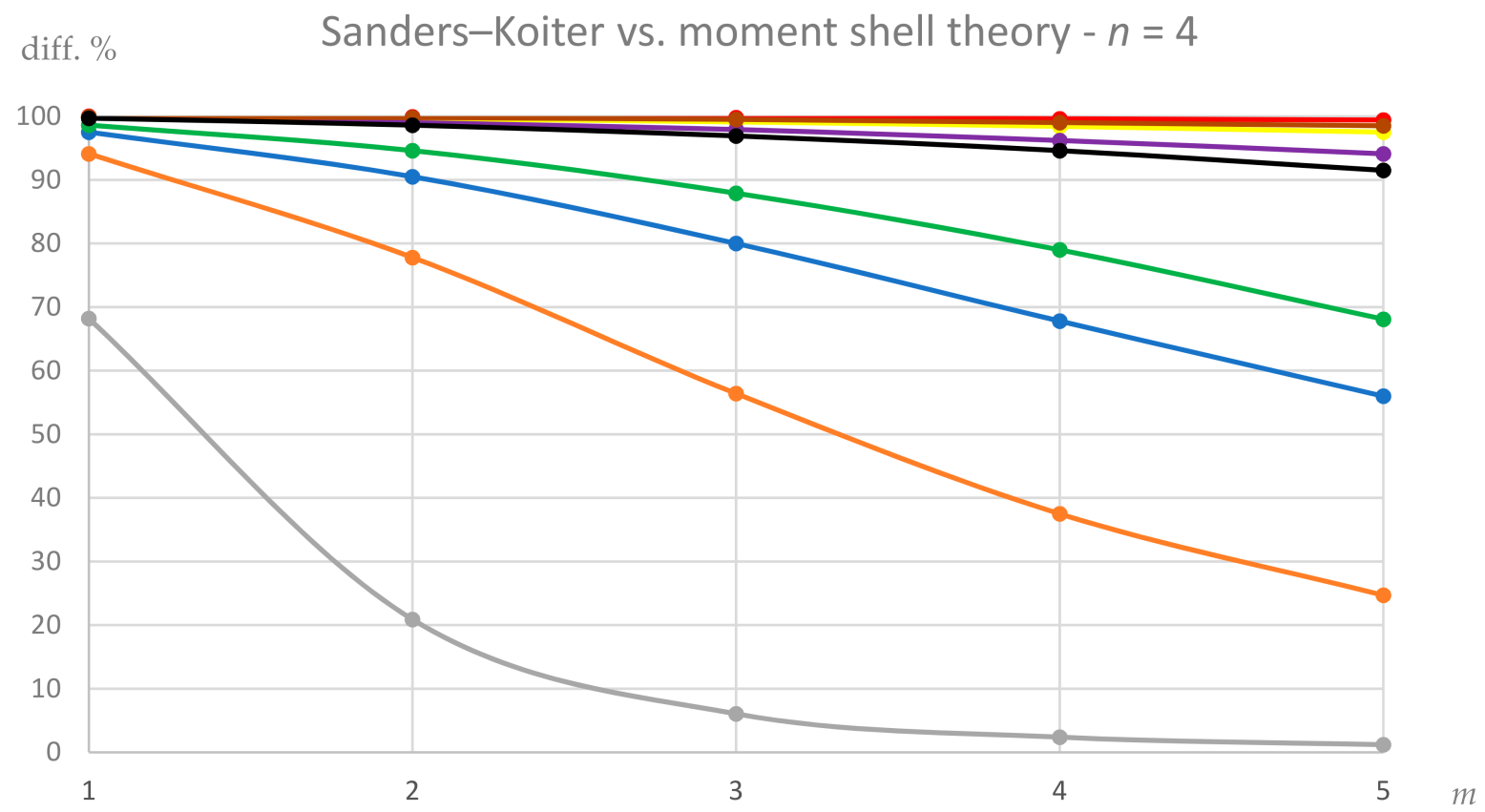

Figure 9. Simply supported DWCNT of Table 1 with different values of aspect ratio $L / R_{2}$ and thickness ratio $R_{2} / h$. Shell-like modes $(n=4)$. Comparisons between extended Sanders-Koiter and simplified moment shell theories, where $L / R_{2}=10$, $R_{2} / h=25 ; \quad L / R_{2}=10, R_{2} / h=50 ; \square L / R_{2}=10, R_{2} / h=250 ; \quad L / R_{2}=50, R_{2} / h=25 ; \quad L / R_{2}=50, R_{2} / h=50 ;$ $L / R_{2}=50, R_{2} / h=250 ; \quad L / R_{2}=100, R_{2} / h=25 ; \quad L / R_{2}=100, R_{2} / h=50 ; \boldsymbol{L} L / R_{2}=100, R_{2} / h=250$.

\section{diff. $\% \quad$ Sanders-Koiter vs. moment shell theory $-n=5$}

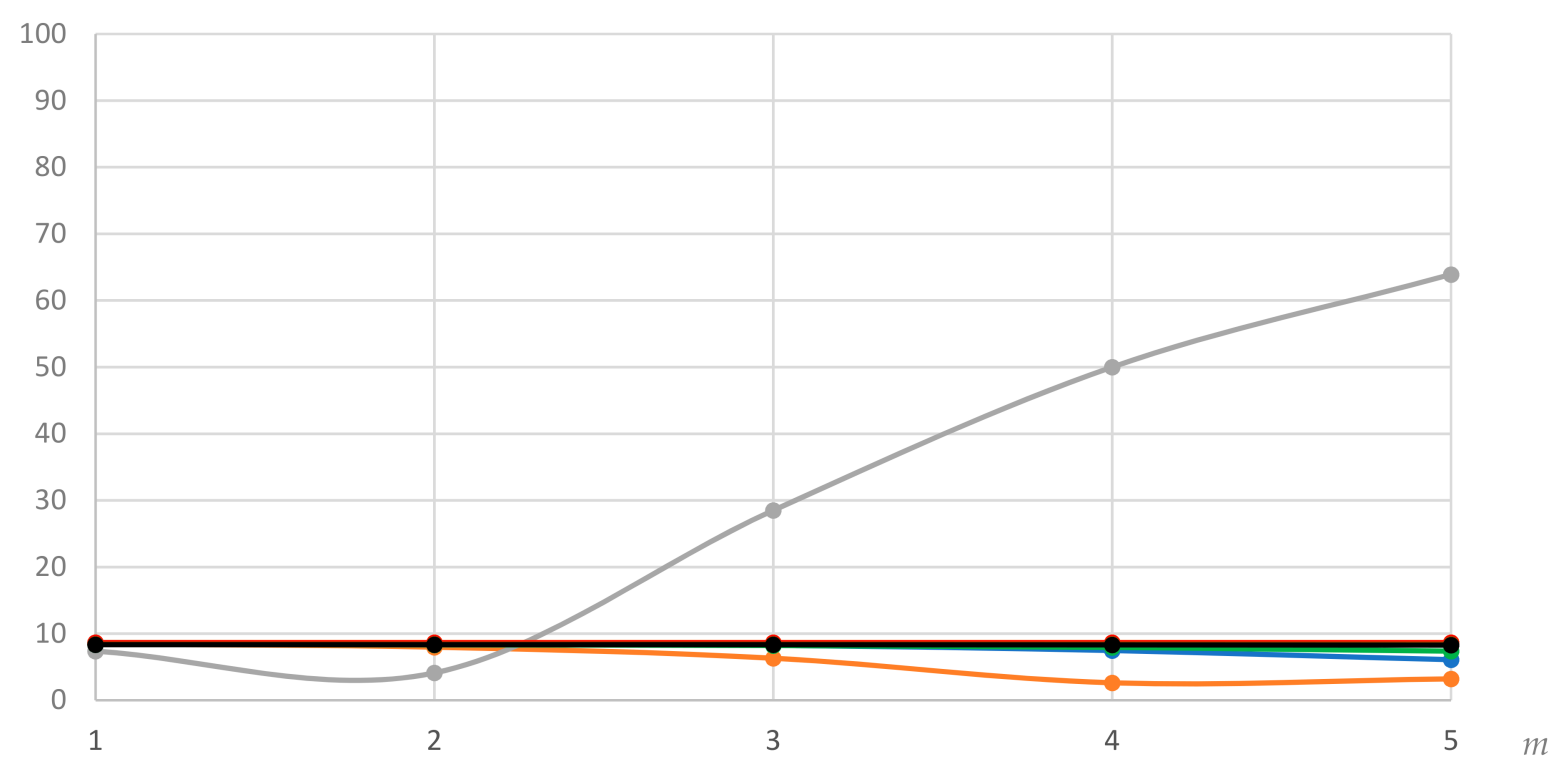

Figure 10. Simply supported DWCNT of Table 1 with different values of aspect ratio $L / R_{2}$ and thickness ratio $R_{2} / h$. Shell-like modes $(n=5)$. Comparisons between extended Sanders-Koiter and simplified moment shell theories, where $L / R_{2}=10, R_{2} / h=25 ; \quad L / R_{2}=10, R_{2} / h=50 ; \quad L / R_{2}=10, R_{2} / h=250 ; \quad L / R_{2}=50, R_{2} / h=25 ; \quad L / R_{2}=50$, $R_{2} / h=50 ; \quad L / R_{2}=50, R_{2} / h=250 ; \quad L / R_{2}=100, R_{2} / h=25 ; \quad L / R_{2}=100, R_{2} / h=50 ; \quad L / R_{2}=100, R_{2} / h=250$. 


\section{diff. $\% \quad$ Sanders-Koiter vs. moment shell theory $-n=6$}

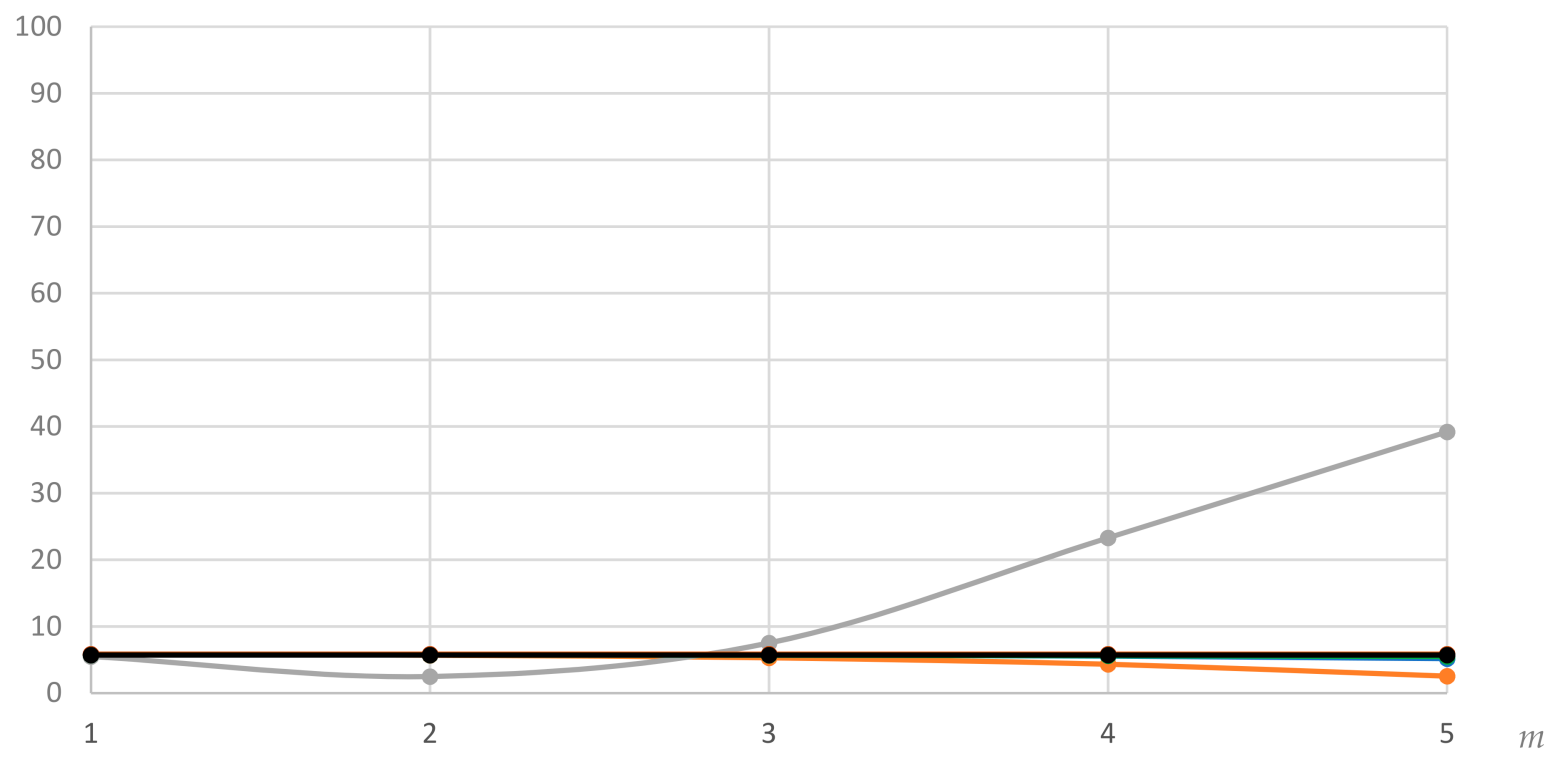

Figure 11. Simply supported DWCNT of Table 1 with different values of aspect ratio $L / R_{2}$ and thickness ratio $R_{2} / h$. Shell-like modes $(n=6)$. Comparisons between extended Sanders-Koiter and simplified moment shell theories, where $L / R_{2}=10, R_{2} / h=25 ; \quad L / R_{2}=10, R_{2} / h=50 ; \quad L / R_{2}=10, R_{2} / h=250 ; \quad L / R_{2}=50, R_{2} / h=25 ; \quad L / R_{2}=50$, $R_{2} / h=50 ; \quad L / R_{2}=50, R_{2} / h=250 ; \quad L / R_{2}=100, R_{2} / h=25 ; \quad L / R_{2}=100, R_{2} / h=50 ; \quad L / R_{2}=100, R_{2} / h=250$.

Therefore, in case of shell-like modes, moment shell theory yields satisfactory results only for very long and relatively thick DWCNTs, when the effect of middle surface strains $\left(\widetilde{\varepsilon}_{x, 0, i}, \widetilde{\varepsilon}_{\theta, 0, i}, \widetilde{\gamma}_{x \theta, 0, i}\right)$ is negligible (very small stretching deformations) and therefore the effect of middle surface changes in curvature and torsion is prevalent $\left(\widetilde{k}_{x, i}, \widetilde{k}_{\theta, i}, \widetilde{k}_{x \theta, i}\right)$ (very high bending/torsional deformations).

Starting from the previous observations, the following results can be obtained:

- $\quad$ by increasing the number of longitudinal half-waves $m$ the percentage difference of the curve $\left(L / R_{2}=10, R_{2} / h=250\right)$ showing maximum percentage difference increases (the contribution of the middle surface strains $\left(\widetilde{\varepsilon}_{x, 0, i}, \widetilde{\varepsilon}_{\theta, 0, i}, \widetilde{\gamma}_{x \theta, 0, i}\right)$ increases);

- by increasing the number of circumferential waves $n$ the percentage difference of the curve $\left(L / R_{2}=10, R_{2} / h=250\right)$ showing maximum percentage difference decreases (the contribution of the middle surface strains $\left(\widetilde{\varepsilon}_{x, 0, i}, \widetilde{\varepsilon}_{\theta, 0, i}, \widetilde{\gamma}_{x \theta, 0, i}\right)$ decreases);

- $\quad$ the maximum percentage difference is obtained at $\left(m=5, n=2, L / R_{2}=10, R_{2} / h=250\right)$ and is equal to 97.6 ;

- $\quad$ by increasing the number of longitudinal half-waves $m$ the percentage difference of the curve $\left(L / R_{2}=100, R_{2} / h=25\right)$ showing maximum percentage difference is approximately constant (the contribution of the middle surface strains $\left(\widetilde{\varepsilon}_{x, 0, i}, \widetilde{\varepsilon}_{\theta, 0, i}, \widetilde{\gamma}_{x \theta, 0, i}\right)$ does not vary);

- $\quad$ by increasing the number of circumferential waves $n$ the percentage difference of the curve $\left(L / R_{2}=100, R_{2} / h=25\right)$ showing minimum percentage difference decreases (the contribution of the middle surface strains $\left(\widetilde{\varepsilon}_{x, 0, i}, \widetilde{\varepsilon}_{\theta, 0, i}, \widetilde{\gamma}_{x \theta, 0, i}\right)$ decreases);

- the minimum percentage difference is obtained at $\left(m=1 \div 5, n=6, L / R_{2}=100\right.$, $\left.R_{2} / h=25\right)$ and is equal to 5.84 .

These results clearly confirm the limitations of membrane and moment shell theories in shell-like vibration modelling of simply supported thin circular cylindrical shells reported in the literature. In particular, in Ventsel [3] it is stated that "the area of application of the membrane theory of cylindrical shells is restricted by fairly short shells $(L / R \ll$ $\sqrt{R / h}$ )" and "the accuracy of the membrane solution decreases with an increase in the shell length". Moreover, in Soedel [4] it is written that the membrane approximation "has some 
justification for shells and arches vibrating in shapes where the stretching of the middle surface is a dominating contributor to motion resistance" (extensional approximation) and "for thickness-to-radius ratios up to approximately $h / R=0.01$ (i.e., for $R / h \geq 100$ ), the membrane approximation gives very good results. Only when the shell starts to become a "thick" shell we see a pronounced effect of bending on the transverse natural frequencies. This is shown for $h / R=0.1$ (i.e., for $R / h \leq 10$ )" (inextensional approximation).

From the computations it was found that, for shell-like modes, similarly to beam-like modes, all middle surface strains are not negligible. Moreover, it was obtained that, in the case of shell-like modes, the middle surface change in curvature along the circumferential direction $\widetilde{k}_{\theta, i}$ increases with increasing the aspect $L / R_{2}$ and thickness $R_{2} / h$ ratios, and so also this term must be taken into account within the model.

Therefore, starting from these results, a new simplified elastic shell model is proposed, based on a combination of the hypotheses of membrane and semi-moment shell theories, neglecting the middle surface terms $\left(\widetilde{k}_{x, i}, \widetilde{k}_{x \theta, i}\right)$ and retaining the middle surface terms $\left(\widetilde{\varepsilon}_{x, 0, i}, \widetilde{\varepsilon}_{\theta, 0, i}, \widetilde{\gamma}_{x \theta, 0, i}, \widetilde{k}_{\theta, i}\right)$ (four terms instead of six) within the expression of the elastic strain energy (12).

In Figures 12-16, comparisons between the extended Sanders-Koiter shell theory and the proposed simplified elastic shell model are reported. It can be observed that:

- for a number of circumferential waves $n=2$, the value of percentage difference is lower than 2 for every DWCNT geometry $\left(L / R_{2}, R_{2} / h\right)$ and number of longitudinal half-waves $m$;

- $\quad$ for $n>2$, the percentage difference of relatively short DWCNTs $\left(L / R_{2}=10\right)$ increases with increasing $m$ and $n$, while the percentage difference of sufficiently long DWCNTs $\left(L / R_{2} \geq 50\right)$ is quasi-constant with increasing $m$ and decreases with increasing $n$, becoming negligible;

- the maximum percentage difference of a relatively short $\left(L / R_{2}=10\right)$ and thick $\left(R_{2} / h=25\right)$ DWCNT is obtained at $(m=5, n=4)$ and is equal to 8.51;

- $\quad$ the maximum percentage difference of a relatively short $\left(L / R_{2}=10\right)$ and thin $\left(R_{2} / h=50\right)$ DWCNT is obtained at $(m=5, n=5)$ and is equal to 6.47 .

\section{diff. \% Sanders-Koiter vs. membrane + semi-moment shell theory $-n=2$}

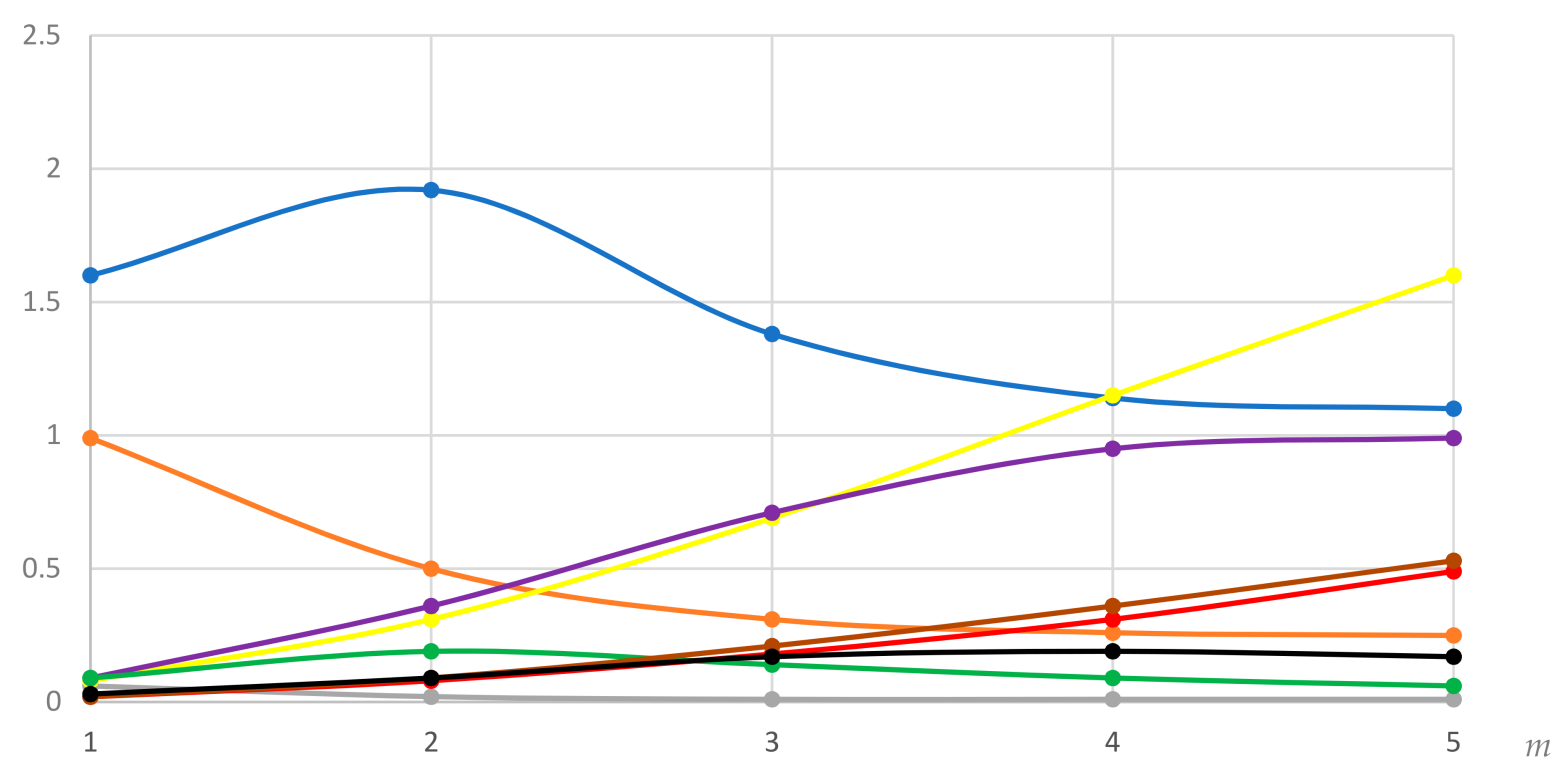

Figure 12. Simply supported DWCNT of Table 1 with different values of aspect ratio $L / R_{2}$ and thickness ratio $R_{2} / h$. Shell-like modes $(n=2)$. Comparisons between extended Sanders-Koiter and membrane + semi-moment shell theory, where $L / R_{2}=10, R_{2} / h=25 ; \quad L / R_{2}=10, R_{2} / h=50 ; \quad L / R_{2}=10, R_{2} / h=250 ; \quad L / R_{2}=50, R_{2} / h=25 ; \quad L / R_{2}=50$, $R_{2} / h=50 ; \quad L / R_{2}=50, R_{2} / h=250 ; \quad L / R_{2}=100, R_{2} / h=25 ; \quad L / R_{2}=100, R_{2} / h=50 ; \quad L / R_{2}=100, R_{2} / h=250$. 


\section{diff. $\%$ Sanders-Koiter vs. membrane + semi-moment shell theory $-n=3$}

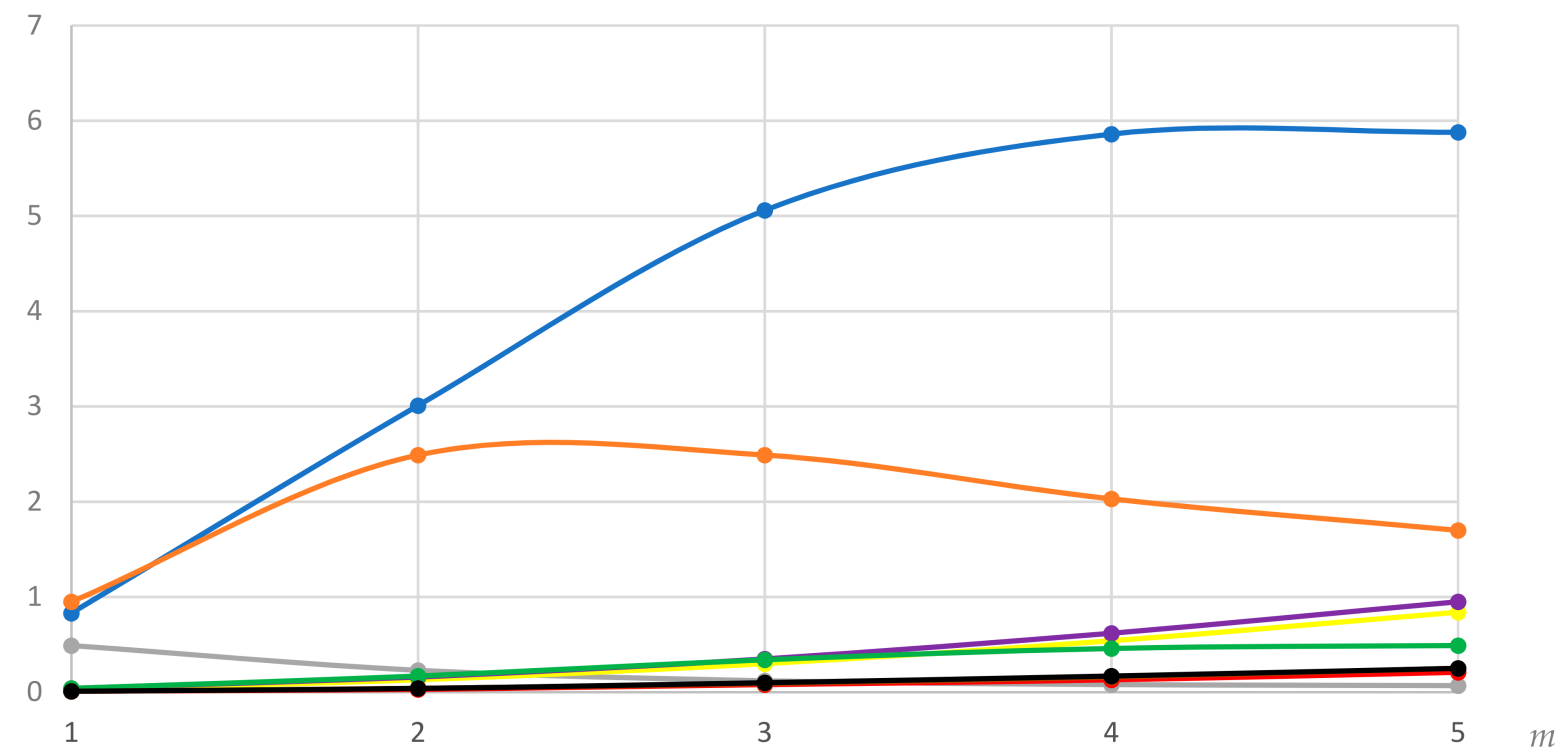

Figure 13. Simply supported DWCNT of Table 1 with different values of aspect ratio $L / R_{2}$ and thickness ratio $R_{2} / h$. Shell-like modes $(n=3)$. Comparisons between extended Sanders-Koiter and membrane + semi-moment shell theory, where $L / R_{2}=10, R_{2} / h=25 ; \quad L / R_{2}=10, R_{2} / h=50 ; \quad L / R_{2}=10, R_{2} / h=250 ; \quad L / R_{2}=50, R_{2} / h=25 ; \quad L / R_{2}=50$, $R_{2} / h=50 ; \quad L / R_{2}=50, R_{2} / h=250 ; \quad L / R_{2}=100, R_{2} / h=25 ; \quad L / R_{2}=100, R_{2} / h=50 ; \quad L / R_{2}=100, R_{2} / h=250$.

\section{diff. $\%$ Sanders-Koiter vs. membrane + semi-moment shell theory $-n=4$}

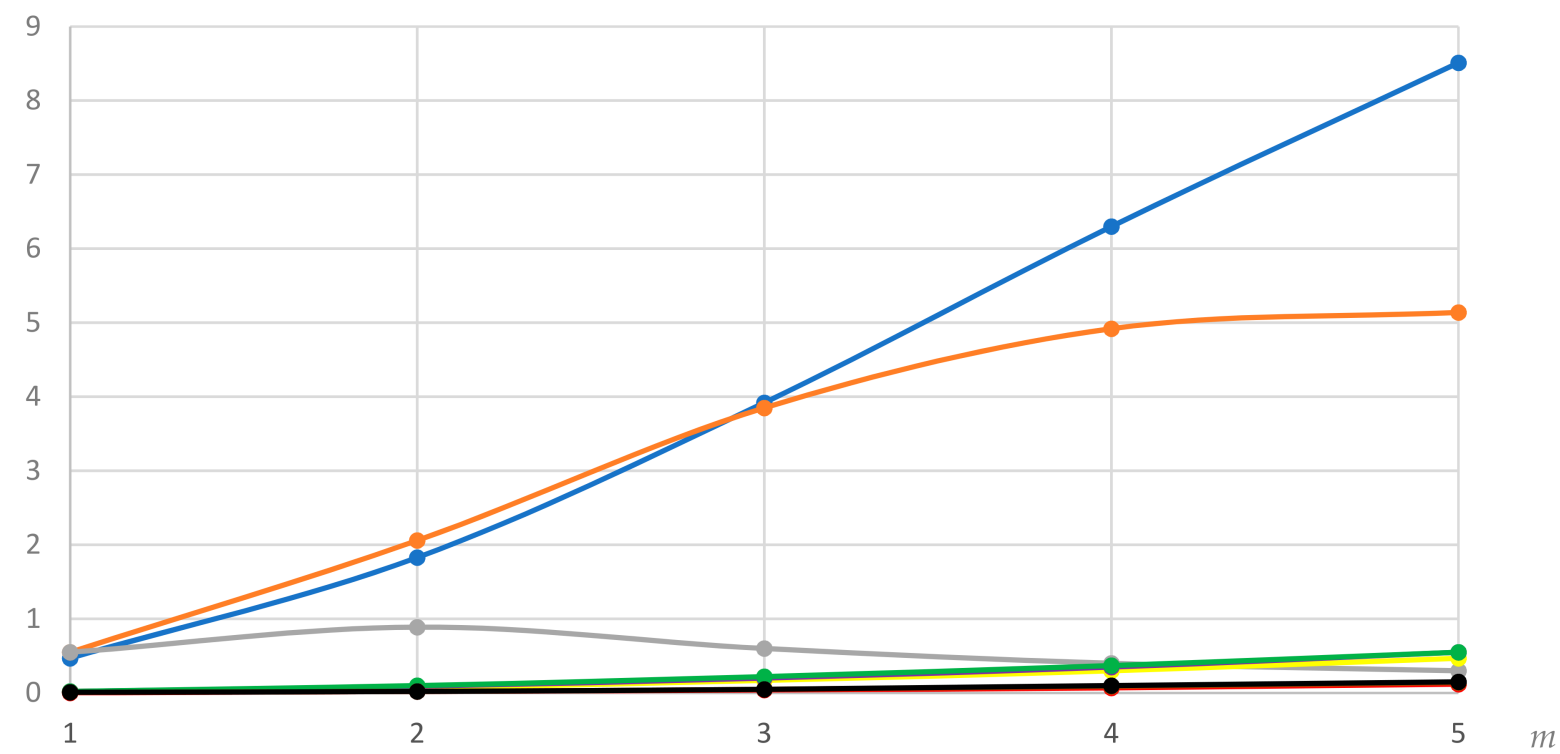

Figure 14. Simply supported DWCNT of Table 1 with different values of aspect ratio $L / R_{2}$ and thickness ratio $R_{2} / h$. Shell-like modes $(n=4)$. Comparisons between extended Sanders-Koiter and membrane + semi-moment shell theory, where $L / R_{2}=10, R_{2} / h=25 ; \quad L / R_{2}=10, R_{2} / h=50 ; \square L / R_{2}=10, R_{2} / h=250 ; \quad L / R_{2}=50, R_{2} / h=25 ; \quad L / R_{2}=50$, $R_{2} / h=50 ;-L / R_{2}=50, R_{2} / h=250 ; \square L / R_{2}=100, R_{2} / h=25 ; \square L / R_{2}=100, R_{2} / h=50 ; \square L / R_{2}=100, R_{2} / h=250$. 
diff. \% Sanders-Koiter vs. membrane + semi-moment shell theory $-n=5$

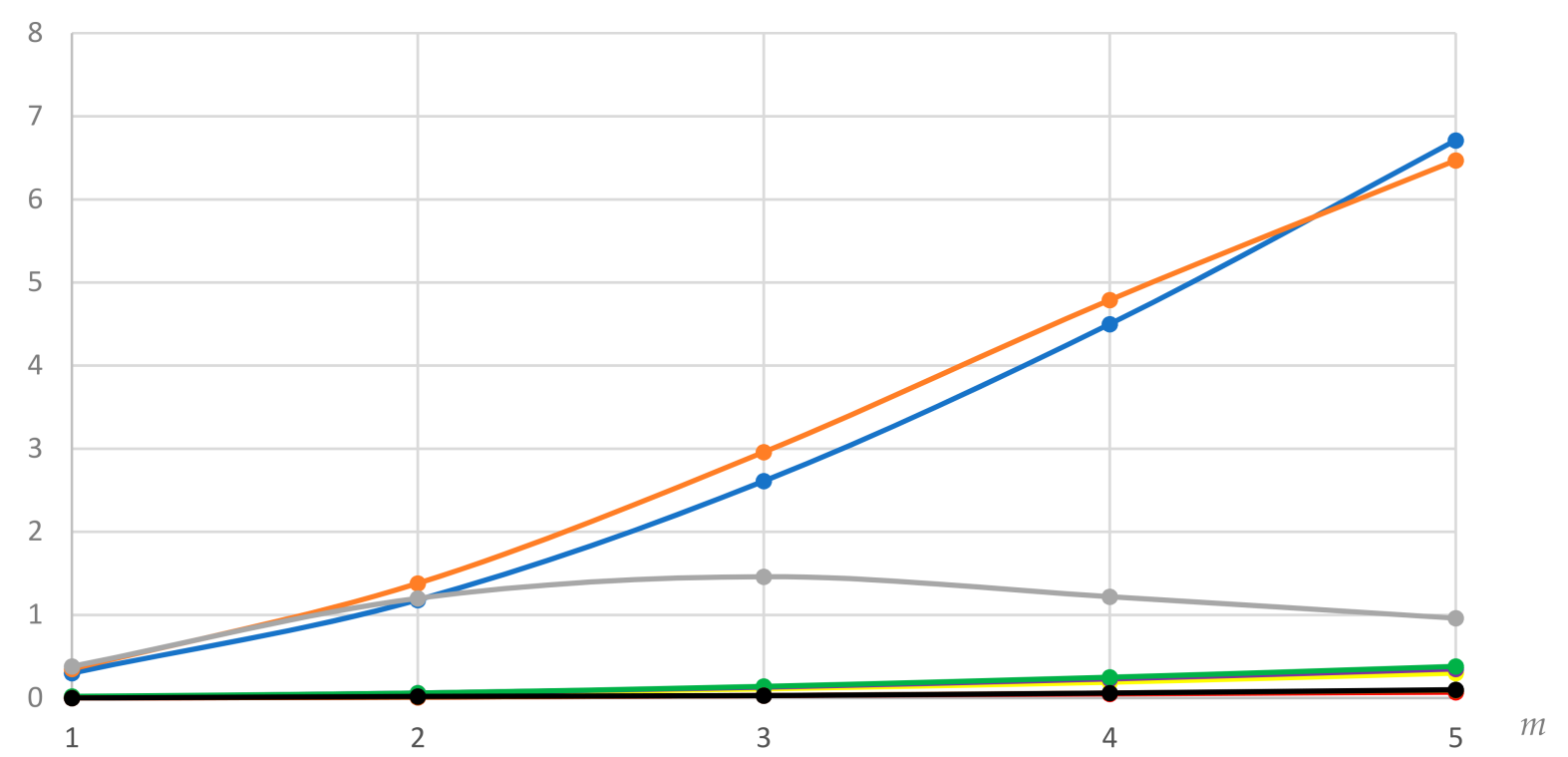

Figure 15. Simply supported DWCNT of Table 1 with different values of aspect ratio $L / R_{2}$ and thickness ratio $R_{2} / h$. Shell-like modes $(n=5)$. Comparisons between extended Sanders-Koiter and membrane + semi-moment shell theory, where $L / R_{2}=10, R_{2} / h=25 ; \quad L / R_{2}=10, R_{2} / h=50 ; \quad L / R_{2}=10, R_{2} / h=250 ; \quad L / R_{2}=50, R_{2} / h=25 ; \quad L / R_{2}=50$, $R_{2} / h=50 ; \quad L / R_{2}=50, R_{2} / h=250 ; \quad L / R_{2}=100, R_{2} / h=25 ; \quad L / R_{2}=100, R_{2} / h=50 ; \quad L / R_{2}=100, R_{2} / h=250$.

\section{diff. $\%$ Sanders-Koiter vs. membrane + semi-moment shell theory $-n=6$}

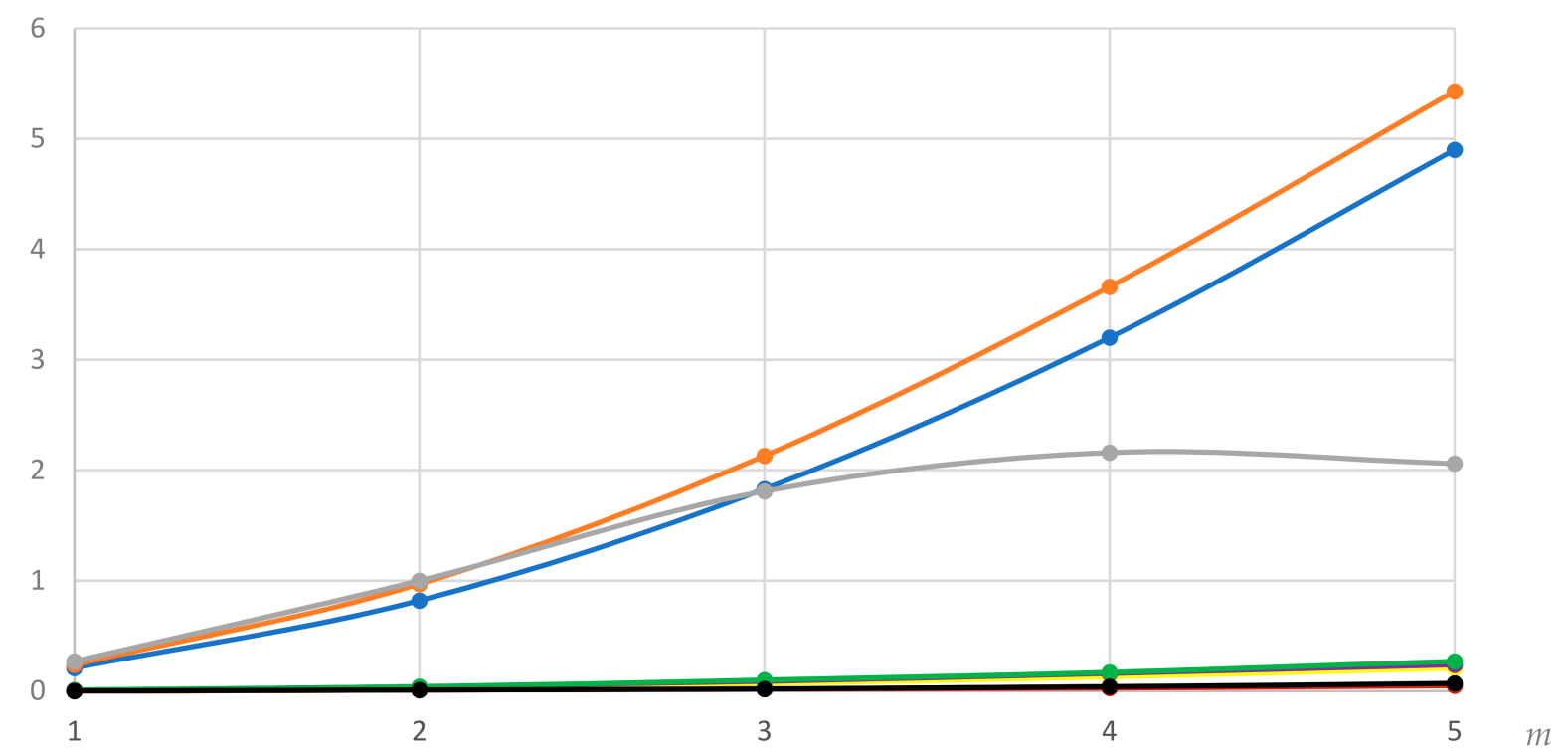

Figure 16. Simply supported DWCNT of Table 1 with different values of aspect ratio $L / R_{2}$ and thickness ratio $R_{2} / h$. Shell-like modes $(n=6)$. Comparisons between extended Sanders-Koiter and membrane + semi-moment shell theory, where $L / R_{2}=10, R_{2} / h=25 ; \quad L / R_{2}=10, R_{2} / h=50 ; \quad L / R_{2}=10, R_{2} / h=250 ; \quad L / R_{2}=50, R_{2} / h=25 ; \quad L / R_{2}=50$, $R_{2} / h=50 ; \quad L / R_{2}=50, R_{2} / h=250 ; \quad L / R_{2}=100, R_{2} / h=25 ; \quad L / R_{2}=100, R_{2} / h=50 ; \quad L / R_{2}=100, R_{2} / h=250$.

In all considered ranges, the new developed simplified elastic shell model, based on a combination of membrane and semi-moment shell theories, provides results in satisfactory agreement and lower computational effort than the extended Sanders-Koiter theory. 
In particular, from the performed simulations, it was found that, for sufficiently long and thin DWCNTs $\left(L / R_{2} \geq 50, R_{2} / h \geq 50\right)$, the contribution of the middle surface change in curvature along the circumferential direction $\widetilde{k}_{\theta, i}$ is strongly prevalent with respect to the ones of the middle surface strains $\left(\widetilde{k}_{\theta, i} \gg\left(\widetilde{\varepsilon}_{x, 0, i}, \widetilde{\varepsilon}_{\theta, 0, i}, \widetilde{\gamma}_{x \theta, 0, i}\right)\right)$.

In addition, from the performed simulations, it was found that, for relatively thick DWCNTs $\left(R_{2} / h=25\right)$, when the effect of the middle surface changes in curvature and torsion is prevalent, $80 \%$ of the difference between extended Sanders-Koiter shell theory and new developed simplified elastic shell model is provided by the middle surface change in torsion $\widetilde{k}_{x \theta, i}$ with respect to the change in curvature along the longitudinal direction $\widetilde{k}_{x, i}$.

\subsection{Characteristic Assumptions of the New Developed Reduced Sanders-Koiter Shell Theory}

Starting from the simplified elastic shell models previously treated, in this section a reduced version of the extended Sanders-Koiter shell theory developed on the basis of the assumptions of the membrane and moment shell theories is reported.

For the axisymmetric modes $(n=0)$, a simplified elastic thin shell model based on the assumptions of the semi-membrane shell theory, which neglects the middle surface terms $\left(\widetilde{\gamma}_{x \theta, 0, i}, \widetilde{k}_{x, i}, \widetilde{k}_{\theta, i}, \widetilde{k}_{x \theta, i}\right)$ and retains the middle surface strains $\left(\widetilde{\varepsilon}_{x, 0, i}, \widetilde{\varepsilon}_{\theta, 0, i}\right)$, is proposed, see Section 4.2.1:

$$
n=0 \quad\left(\widetilde{\gamma}_{x \theta, 0, i}, \widetilde{k}_{x, i}, \widetilde{k}_{\theta, i}, \widetilde{k}_{x \theta, i}\right)=0 \quad\left(\widetilde{\varepsilon}_{x, 0, i}, \widetilde{\varepsilon}_{\theta, 0, i}\right) \neq 0
$$

For the beam-like modes $(n=1)$, a simplified elastic thin shell model based on the assumptions of the membrane shell theory, which neglects the middle surface changes in curvature and torsion of the shell $\left(\widetilde{k}_{x, i}, \widetilde{k}_{\theta, i}, \widetilde{k}_{x \theta, i}\right)$ and retains the middle surface strains $\left(\widetilde{\varepsilon}_{x, 0, i}, \widetilde{\varepsilon}_{\theta, 0, i}, \widetilde{\gamma}_{x \theta, 0, i}\right)$, is proposed, see Section 4.2.2:

$$
n=1 \quad\left(\widetilde{k}_{x, i}, \widetilde{k}_{\theta, i}, \widetilde{k}_{x \theta, i}\right)=0 \quad\left(\widetilde{\varepsilon}_{x, 0, i}, \widetilde{\varepsilon}_{\theta, 0, i}, \widetilde{\gamma}_{x \theta, 0, i}\right) \neq 0
$$

For the shell-like modes $(n \geq 2)$, a new developed simplified elastic thin shell model based on a combination of the assumptions of the membrane and semi-moment shell theory, which neglects the middle surface changes in curvature and torsion $\left(\widetilde{k}_{x, i}, \widetilde{k}_{x \theta, i}\right)$ and retains the middle surface terms $\left(\widetilde{\varepsilon}_{x, 0, i}, \widetilde{\varepsilon}_{\theta, 0, i}, \widetilde{\gamma}_{x \theta, 0, i}, \widetilde{k}_{\theta, i}\right)$, is proposed, see Section 4.2.3:

$$
n \geq 2 \quad\left(\widetilde{k}_{x, i}, \widetilde{k}_{x \theta, i}\right)=0 \quad\left(\widetilde{\varepsilon}_{x, 0, i}, \widetilde{\varepsilon}_{\theta, 0, i}, \widetilde{\gamma}_{x \theta, 0, i}, \widetilde{k}_{\theta, i}\right) \neq 0
$$

The reduced Sanders-Koiter shell theory reported is a simplified elastic shell model that is applicable for linear vibrations of DWCNTs (with maximum relative error around $8 \%$ ) in a very large range of geometries and numbers of waves, including some cases in which membrane and moment shell models result in much larger relative errors.

These results are very significant for further applications to CNT vibrations because simplified elastic shell models, which are characterized by a lower number of middle surface components in the expression of the elastic strain energy, and therefore a lower computational effort into the numerical simulations, with respect to the extended elastic shell models (e.g., the extended Sanders-Koiter shell theory), result particularly useful for linear (and even more nonlinear) vibrations of MWCNTs with large number of layers.

On the other hand, it must be stressed that these simplified elastic shell models are applicable only for radial deflection of thin cylindrical shells in the presence of simple boundary conditions (e.g., simply supported edges). When longitudinal or circumferential deflections become essential, or more complicated boundary conditions are applied, then more accurate elastic shell models (e.g., extended Sanders-Koiter shell theory) should be adopted, see Refs. [3,4,7] for more details. 


\section{Conclusions}

Applicability and limitations of simplified models of thin elastic circular cylindrical shells for linear vibrations of double-walled carbon nanotubes (DWCNTs) with comparison to the extended Sanders-Koiter shell theory are studied. Different values of aspect and thickness ratios, and different numbers of waves along the longitudinal and circumferential directions, are analysed. In case of axisymmetric modes it is found that a reduced form of the membrane shell theory, also neglecting the tangential shear strain, gives excellent agreement with the extended Sanders-Koiter shell theory. Again, for beam-like modes, it is demonstrated that membrane shell theory gives excellent agreement with the extended Sanders-Koiter shell theory. As for shell-like modes it is found that both membrane and moment shell theories are not in acceptable agreement with the extended Sanders-Koiter shell theory for a large range of DWCNT aspect and thickness ratios. Therefore, in case of shell-like modes, a new simplified elastic shell model, based on the combination of membrane and semi-moment theories and neglecting middle surface changes in curvature along the axial direction and torsion, is developed, providing satisfactory agreement with the extended Sanders-Koiter shell theory. By considering the semi-membrane shell theory for axisymmetric modes, the membrane shell theory for beam-like modes and the combination of membrane and semi-moment theories for shell-like modes, it is obtained a reduced version of the Sanders-Koiter shell theory that presents a significantly enlarged range of applicability compared with both membrane and moment shell theories, giving results with excellent, or at least acceptable, agreement for all geometries and numbers of waves with respect to the extended Sanders-Koiter shell theory.

Author Contributions: Conceptualization, M.S. and I.E.E.; methodology, M.S. and I.E.E.; supervision: O.V.G. and F.P.; writing — original draft preparation, M.S. and I.E.E.; writing — review and editing, O.V.G. and F.P. All authors have read and agreed to the published version of the manuscript.

Funding: This research was funded by Israel Science Foundation, grant number 1696/17 (authors Matteo Strozzi and Oleg V. Gendelman) and Edmund Safra Foundation, grant number 2018 (author Isaac E. Elishakoff).

Institutional Review Board Statement: Not applicable.

Acknowledgments: This work is dedicated to the blessed memory of Leonid I. Manevitch, outstanding educator, respected teacher, admired scholar, who died, after severe illness, on 20 August 2020, at the age of 82. Manevitch followed the research presented in this paper with great attention to the end, dispensing useful suggestions on mathematical modelling and pointing out interesting comparative works in literature. In previously published works Manevitch investigated applicability and limitations of simplified elastic shell theories for vibration modelling of SWCNTS: the Authors of the present work extended these relevant studies to DWCNTs with the perspective of investigate linear and nonlinear vibrations of MWCNTs.

Conflicts of Interest: The authors declare no conflict of interest.

\section{References}

1. Buczkowski, W.; Szymczak-Graczyk, A.; Walczak, Z. Experimental validation of numerical static calculations for a monolithic rectangular tank with walls of trapezoidal cross-section. Bull. Pol. Acad. Sci. Tech. Sci. 2017, 65, 799-804. [CrossRef]

2. Szymczak-Graczyk, A. The Effect of Subgrade Coefficient on Static Work of a Pontoon Made as a Monolithic Closed Tank. Appl. Sci. 2021, 11, 4259. [CrossRef]

3. Ventsel, E.; Krauthammer, T. Thin Plates and Shells. In Theory, Analysis, and Applications; The Pennsylvania State University, Marcel Dekker: New York, NY, USA, 2001.

4. Soedel, W. Vibrations of Shells and Plates, 3rd ed.; Marcel Dekker: New York, NY, USA, 2004.

5. Calladine, C. Theory of Shell Structures; Cambridge University Press: New York, NY, USA, 1983.

6. Yamaki, N. Elastic Stability of Circular Cylindrical Shells; Elsevier: Amsterdam, The Netherlands, 1984.

7. Leissa, A.W. Vibration of Shells; Acoustical Society of America: Columbus, OH, USA, 1993.

8. Amabili, M. Nonlinear Vibrations and Stability of Shells and Plates; Cambridge University Press: New York, NY, USA, 2008.

9. Love, A.E.H. A Treatise on the Mathematical Theory of Elasticity, 4th ed.; Dover: New York, NY, USA, 1944.

10. Green, A.E. On the Linear Theory of Thin Elastic Shells. Proc. R. Soc. Lond. Ser. A Math. Phys. Sci. 1962, 266, 143-160.

11. Reissner, E. Stress strain relations in the theory of thin elastic shells. J. Math. Phys. 1952, 31, 109-119. [CrossRef] 
12. Sanders, J.L. An Improved First Approximation Theory for Thin Shells; NASA TR-R24; U.S. Government Printing Office: Washington, DC, USA, 1959.

13. Koiter, W.T. A consistent First Approximation in the General Theory of Thin Elastic Shells. In Proceedings of the Symposium on Theory of Thin Elastic Shells, Delft, The Netherlands, 24-28 August 1959.

14. Donnell, L.H. Beams, Plates, and Shells; McGraw-Hill: New York, NY, USA, 1976.

15. Vlasov, V.Z. General Theory of Shells and Its Applications in Engineering; NASA Technical Translation F-99: Washington, DC, USA, 1964.

16. Mushtari, K.M. Certain generalizations of the theory of thin shells. Izv. Fiz. Mat. Ob-Va. Pri Kaz. Un-Te 1938, 11, 28-56. (In Russian)

17. Timoshenko, S.; Woinowsky-Krieger, S. Theory of Plates and Shells; McGraw-Hill: New York, NY, USA, 1959.

18. Naghdi, P.M. A survey on resent progress in the theory of thin elastic shells. Appl. Mech. Rev. 1956, 9, 365-368.

19. Lur'ye, A.I. General theory of elastic shells. Prikl. Mat. Mekh. 1940, 4, 7-34. (In Russian)

20. Flugge, W. Stresses in Shells, 2nd ed.; Springer: Berlin/Heidelberg, Germany, 1962.

21. Byrne, R. Theory of Small Deformations of a Thin Elastic Shell; Seminar Reports in Mathematics; University of California: Berkeley, CA, USA, 1944; Volume 2, pp. 103-152.

22. Novozhilov, V.V. Thin Shell Theory; Springer: Groningen, The Netherlands, 1964. (In Russian)

23. Gol'denveizer, A.L. Theory of Elastic Thin Shells; Pergamon Press: New York, NY, USA, 1961.

24. Reissner, E. A new derivation of the equations of the deformation of elastic shells. Am. J. Math. 1941, 63, 177-184. [CrossRef]

25. Sokolovskii, V.V. Momentless shells of revolution. Prikl. Mat. Mekh. 1938, 1, 81-88. (In Russian)

26. Truesdell, C. The Membrane Theory of Shells of Revolution. Trans. Am. Math. Soc. 1945, 58, 96-166. [CrossRef]

27. Gibson, J.E. Thin Shells. Computing and Theory; Pergamon Press: New York, NY, USA, 1980.

28. Gibson, J.E. Linear Elastic Theory of Thin Shells; Pergamon Press: New York, NY, USA, 1965.

29. Elishakoff, I.; Pentaras, D.; Dujat, K.; Versaci, C.; Muscolino, G.; Wang, C.M.; Ghyselinck, G. Carbon Nanotubes and Nano Sensors: Vibrations, Buckling, and Ballistic Impact; John Wiley \& Sons: London, UK, 2012.

30. Liew, K.M.; Jianwei, Y.; Zhang, L. Mechanical Behaviors of Carbon Nanotubes: Theoretical and Numerical Approaches; Elsevier: Amsterdam, The Netherlands, 2017.

31. Tserpes, K.I.; Silvestre, N. Modeling of Carbon Nanotubes, Graphene and Their Composites; Springer International Publishing: Cham, Switzerland, 2014.

32. Harik, V. Trends in Nanoscale Mechanics: Mechanics of Carbon Nanotubes, Graphene, Nanocomposites and Molecular Dynamics; Springer: Dordrecht, The Netherlands, 2014.

33. Rahman, A.; Ali, I.; Al Zahrani, S.M.; Eleithy, R.H. A review of the applications of nanocarbon polymer composites. NANO Brief Rep. Rev. 2011, 6, 185-203. [CrossRef]

34. Thostenson, E.T.; Ren, Z.; Chou, T.W. Advances in the science and technology of carbon nanotubes and their composites: A review. Compos. Sci. Technol. 2001, 61, 1899-1912. [CrossRef]

35. Jensen, B.D.; Kim, J.W.; Sauti, G.; Wise, K.E.; Dong, L.; Wadley, H.N.G.; Park, J.G.; Liang, R.; Siochi, E.J. Toward ultralight high-strength structural materials via collapsed carbon nanotube bonding. Carbon 2020, 156, 538-548. [CrossRef]

36. Al-Ghalith, J.; Xu, H.; Dumitrica, T. Collapsed carbon nanotubes as building blocks for high-performance thermal materials. Phys. Rev. Mater. 2017, 1, 056001. [CrossRef]

37. Zhang, S.; Ma, Y.; Suresh, L.; Hao, A.; Bick, M.; Tan, S.C.; Chen, J. Carbon Nanotube Reinforced Strong Carbon Matrix Composites. Am. Chem. Soc. ACS Nano 2020, 14, 9282-9319. [CrossRef]

38. Rao, A.M.; Richter, E.; Bandow, S.; Chase, B.; Eklund, P.C.; Williams, K.A.; Fang, S.; Subbaswamy, K.R.; Menon, M.; Thess, A.; et al. Diameter-selective Raman scattering from vibrational modes in carbon nanotubes. Science 1997, 275, 187-191. [CrossRef]

39. Bandow, S.; Asaka, S.; Saito, Y.; Rao, A.M.; Grigorian, L.; Richter, E.; Eklund, P.C. Effect of the growth temperature on the diameter distribution and chirality of single-wall carbon nanotubes. Phys. Rev. Lett. 1998, 80, 3779-3782. [CrossRef]

40. Jorio, A.; Saito, R.; Hafner, J.H.; Lieber, C.M.; Hunter, M.; McClure, T.; Dresselhaus, G.; Dresselhaus, M.S. Structural (n,m) determination of isolated single-wall carbon nanotubes by resonant Raman scattering. Phys. Rev. Lett. 2001, 86, 1118-1121. [CrossRef]

41. Gupta, S.S.; Bosco, F.G.; Batra, R.C. Breakdown of structural models for vibrations of single-wall zigzag carbon nanotubes. J. Appl. Phys. 2009, 106, 063527. [CrossRef]

42. Cheng, H.C.; Liu, Y.L.; Wu, C.H.; Chen, W.H. On radial breathing vibration of carbon nanotubes. Comput. Methods Appl. Mech. Eng. 2010, 199, 2820-2827. [CrossRef]

43. Gupta, S.S.; Bosco, F.G.; Batra, R.C. Wall thickness and elastic moduli of single-walled carbon nanotubes from frequencies of axial, torsional and inextensional modes of vibration. Comput. Mater. Sci. 2010, 47, 1049-1059. [CrossRef]

44. Qian, D.; Wagner, G.J.; Liu, W.K.; Yu, M.F.; Ruoff, R.S. Mechanics of Carbon Nanotubes. Appl. Mech. Rev. 2002, 55, 495-533. [CrossRef]

45. Huang, Y.; Wang, Z. Mechanics of Nanotubes, Comprehensive Structural Integrity Handbook; Elsevier: New York, NY, USA, 2003; Volume 8, pp. 551-579.

46. Yakobson, B.I.; Brabec, C.J.; Bernholc, J. Nanomechanics of Carbon Tubes: Instability Beyond Linear Response. Phys. Rev. Lett. 1996, 76, 2511-2514. [CrossRef]

47. Ru, C.Q. Effective Bending Stiffness of Carbon Nanotubes. Phys. Rev. B 2000, 62, 9973-9976. [CrossRef] 
48. Ru, C.Q. Degraded Axial Buckling Strain of Multiwalled Carbon Nanotubes Due to Interlayer Slips. J. Appl. Phys. 2001, 89, 3426-3433. [CrossRef]

49. Liew, K.; Wang, Q. Analysis of wave propagation in carbon nanotubes via elastic shell theories. Int. J. Eng. Sci. 2007, 45, 227-241. [CrossRef]

50. Ru, C.Q. Axially compressed buckling of a double-walled carbon nanotube embedded in an elastic medium. J. Mech. Phys. Solids 2001, 49, 1265-1279. [CrossRef]

51. He, X.Q.; Eisenberger, M.; Liew, K.M. The effect of Van der Waals interaction modeling on the vibration characteristics of multiwalled carbon nanotubes. J. Appl. Phys. 2006, 100, 124317. [CrossRef]

52. Elishakoff, I.; Pentaras, D. Fundamental natural frequencies of double-walled carbon nanotubes. J. Sound Vib. 2009, 322, 652-664. [CrossRef]

53. Silvestre, N.; Wang, C.M.; Zhang, Y.Y.; Xiang, Y. Sanders shell model for buckling of single-walled carbon nanotubes with small aspect ratio. Compos. Struct. 2011, 93, 1683-1691. [CrossRef]

54. Silvestre, N. On the accuracy of shell models for torsional buckling of carbon nanotubes. Eur. J. Mech. A/Solids 2012, 32, 103-108. [CrossRef]

55. Wang, C.Y.; Ru, C.Q.; Mioduchowski, A. Applicability and Limitations of Simplified Elastic Shell Equations for Carbon Nanotubes. J. Appl. Mech. 2004, 71, 622-631. [CrossRef]

56. Ghavanloo, E.; Fazelzadeh, S.A. Vibration characteristics of single-walled carbon nanotubes based on anisotropic elastic shell model including chirality effect. Appl. Math. Model. 2012, 36, 4988-5000. [CrossRef]

57. Strozzi, M.; Smirnov, V.V.; Manevitch, L.I.; Pellicano, F. Nonlinear vibrations and energy exchange of single-walled carbon nanotubes. Radial breathing modes. Compos. Struct. 2018, 184, 613-632. [CrossRef]

58. Strozzi, M.; Smirnov, V.V.; Manevitch, L.I.; Milani, M.; Pellicano, F. Nonlinear vibrations and energy exchange of single-walled carbon nanotubes. Circumferential flexural modes. J. Sound Vib. 2016, 381, 156-178. [CrossRef]

59. Strozzi, M.; Pellicano, F. Nonlinear Resonance Interaction between Conjugate Circumferential Flexural Modes in Single-Walled Carbon Nanotubes. Shock Vib. 2019, 2019, 3241698. [CrossRef]

60. Strozzi, M.; Smirnov, V.V.; Manevitch, L.I.; Pellicano, F. Nonlinear normal modes, resonances and energy exchange in single-walled carbon nanotubes. Int. J. Non-Linear Mech. 2020, 120, 103398. [CrossRef]

61. Strozzi, M.; Pellicano, F. Linear vibrations of triple-walled carbon nanotubes. Math. Mech. Solids 2018, 23, 1456-1481. [CrossRef]

62. Strozzi, M.; Manevitch, L.I.; Pellicano, F.; Smirnov, V.V.; Shepelev, D.S. Low-frequency linear vibrations of single-walled carbon nanotubes: Analytical and numerical models. J. Sound Vib. 2014, 333, 2936-2957. [CrossRef]

63. Kaplunov, J.; Manevitch, L.I.; Smirnov, V.V. Vibrations of an elastic cylindrical shell near the lowest cut-off frequency. Proc. R. Soc. A 2018, 472, 20150753. [CrossRef]

64. Andrianov, I.; Kaplunov, J.; Kudaibergenov, A.; Manevitch, L. The effect of a weak nonlinearity on the lowest cut-off frequencies of a cylindrical shell. Z. Fur Angew. Math. Und Phys. 2018, 9, 1-12. [CrossRef] 